\title{
NONLINEAR FILTERING FOR OBSERVATIONS ON A RANDOM VECTOR FIELD ALONG A RANDOM PATH. APPLICATION TO ATMOSPHERIC TURBULENT VELOCITIES
}

\author{
Christophe Baehr ${ }^{1,2}$
}

\begin{abstract}
To filter perturbed local measurements on a random medium, a dynamic model jointly with an observation transfer equation are needed. Some media given by PDE could have a local probabilistic representation by a Lagrangian stochastic process with mean-field interactions. In this case, we define the acquisition process of locally homogeneous medium along a random path by a Lagrangian Markov process conditioned to be in a domain following the path and conditioned to the observations. The nonlinear filtering for the mobile signal is therefore those of an acquisition process contaminated by random errors. This will provide a Feynman-Kac distribution flow for the conditional laws and an $N$ particle approximation with a $\mathcal{O}\left(\frac{1}{\sqrt{N}}\right)$ asymptotic convergence. An application to nonlinear filtering for 3D atmospheric turbulent fluids will be described.
\end{abstract}

Mathematics Subject Classification. 82B31, 65C35, 65C05, 62M20, 60G57, 60J85.

Received May 20, 2009. Revised December 23, 2009.

Published online August 26, 2010.

\section{INTRODUCTION}

In Applied Sciences the knowledge of a (physical, biological, etc.) state system needs measurements. Often it is not possible to recover the measured values everywhere in the state space but only on discrete points or along a path or barely on a grid. Our interest is focused on the case of measurements along a random path in a random medium. The other situations can be considered as a reduction or combinations of this case. This paper is devoted to the definition of a new process called Acquisition Process and using this process to nonlinear filtering of corrupted measurements of a stochastic vector fields made along a random path. The physical application concerns measurements of atmospheric turbulent fluids with a mobile sensor.

Stochastic nonlinear filtering requires a model for the signal to be denoised. If the studied system can be represented as a set of equations, global or local, for mobile measurements into this system there are in general no model. We propose here a method using a local model of the random medium to derive a dynamic model for mobile measurements signal.

\footnotetext{
Keywords and phrases. Nonlinear filtering, Feynman-Kac, stochastic model, turbulence.

${ }^{1}$ Météo-France-CNRS, CNRM-GAME URA1357, 42 avenue Coriolis, 31057 Toulouse Cedex 1, France.

christophe.baehr@meteo.fr

2 Associated member of the Laboratory of Statistics and Probability of the Toulouse Mathematics Institute (UMR 5219), 118 route de Narbonne, 31062 Toulouse Cedex 9, France.
} 
The representation of the solution of partial differential equations by the expectation of a stochastic process is well known for linear parabolic equation (see [12]). For nonlinear partial differential equations it is not so clear. Without a general theory sometimes it needs particle systems to have an approximated solution [13]. In the particular case of the Navier-Stokes equation, there is nonlinear McKean-Vlasov equations to represent the small scale dynamics as random walks. Some are exact but need implicit calculations ([7] or [6]), some have uniqueness and existence proved only for the dimension 2 ([11] or [14]), some are phenomenological but able to approach 3D inhomogeneous flows [15] or stratified flows [8].

The measurements along a path are local processes, living on a line. This kind of measurements do not allow the reconstruction of the entire observed field. In Section 1, using a local stochastic representation of the medium and the known sensor trajectory, we will define a process and will give (Sect. 2) a procedure to estimate locally the parameters of the medium. Then to denoise the measurements corrupted by instruments, we suggest a filtering algorithm to compute the conditional distribution of states knowing partial observations. By the physical context under consideration, this filter have to deal with mean-field processes, indeed the local estimations are connected with large scale structures, and these connections will be made through the average of large scale fields. Particle filter are well adapted to compute at the same time the filtering conditional laws and the mean-field terms. We propose in Section 3 some algorithmic solutions to solve this probabilist estimation problems.

Application to measurements of atmospheric wind will be presented in Section 4. This application will use all the material presented in the previous sections and a particular representation of the Navier-Stokes equations adapted to the atmospheric physics.

\section{ACQUisition PROCESS}

In the problem of the examination of a multidimensional field along a path, there is a clear separation between the trajectories and the other components of the state space. Then we consider the phase space as the Cartesian product $E \times E^{\prime}$ where $E$ is the configuration space where lie the trajectories and $E^{\prime}$ the vector space. Now we need some definitions. They could have a very wide range of uses and we will detail few of them.

Definition 1.1 (Acquisition System). Let $E \subset \mathbb{R}^{d}, d \in \mathbb{N}^{*}$, be a metric locally compact space of point called configuration space. $E$ is endowed with the $\sigma$-algebra $\mathcal{E}$.

Let $E^{\prime} \subset \mathbb{R}^{d^{\prime}}, d^{\prime} \in \mathbb{N}^{*}$, be a vector space called phase space endowed with the $\sigma$-algebra $\mathcal{E}^{\prime}$. Let $\left(\Omega, \mathcal{F},\left(\mathcal{F}_{t}\right)_{t \geq 0}, \mathbb{P}\right)$ be a complete filtered probability space.

Let $T<\infty$ be a real number and $x \in E$ a point of the configuration space. Let $X_{t}$ be the $(E, \mathcal{E})$-valued random variable family on $\left(\Omega, \mathcal{F}, \mathcal{F}_{t}\right)$ indexed by the time $t \in[0, T]$ and $X_{t, x}^{\prime}$ be the $\left(E^{\prime}, \mathcal{E}^{\prime}\right)$-valued random variable family on $\left(\Omega, \mathcal{F}, \mathcal{F}_{t}\right)$ indexed by the time $t \in[0, T]$ and the point $x \in E$.

Then, the pair of applications $\mathcal{F}_{t}$-measurable, $\left(X_{t}, X_{t, x}^{\prime}\right)$ is called the Acquisition System of the random vector field.

The process $X_{t}$ is called the Acquisition Path and the family $X_{t, x}^{\prime}$ is called the Acquisition Field.

This is the choice of the path process $X_{t}$, the Acquisition Field $X_{t, x}^{\prime}$ and their possible coupling which makes sense to the Acquisition System and defines the Acquisition Process which is the statement of the field $X_{t, x}^{\prime}$ along the path $X_{t}$.

Definition 1.2 (Acquisition Process). Let $\left(X_{t}, X_{t, x}^{\prime}\right)$ be a $\left((E, \mathcal{E}) \times\left(E^{\prime}, \mathcal{E}^{\prime}\right)\right)$-valued Acquisition System on the probability space $\left(\Omega, \mathcal{F}, \mathcal{F}_{t}, \mathbb{P}\right)$. For all $t \in[0, T]$, the Acquisition Process is defined by the $\left(E^{\prime}, \mathcal{E}^{\prime}\right)$-valued process on $\left(\Omega, \mathcal{F}, \mathcal{F}_{t}\right) A_{t}$ with

$$
A_{t} \stackrel{\text { def }}{=} X_{t, X_{t}}^{\prime}
$$

Example 1.1. Let $x$ be a fixed point in $E$. For all $t>0$ we consider the stationary acquisition path $X_{t}=x$. Then the acquisition system is $\left(x, X_{t, x}^{\prime}\right)$ and the acquisition process is $A_{t}=X_{t, x}^{\prime}$ which can be called, by analogy with physical applications, the Eulerian Acquisition of the field. 
Example 1.2. Let $X_{t, x}^{\prime}$ be a $\left(E^{\prime}, \mathcal{E}^{\prime}\right)$-valued random bounded vector field $\mathcal{C}^{\infty}$. Let $x_{0}$ be a point in $E$ and for all $0 \leq t \leq T$, let $W_{t}$ be a $\mathcal{F}_{t}$-Brownian Motion on $\left(\Omega, \mathcal{F}, \mathcal{F}_{t}, \mathbb{P}\right)$. We define the stochastic flow (see [4]):

$$
X_{t}^{x_{0}}=x_{0}+\int_{0}^{t} X_{s, X_{s}^{x_{0}}}^{\prime} \circ \mathrm{d} W_{s}
$$

where o signs the Stratonovich integral.

Then the Acquisition Process is $A_{t}=X_{t, X_{t}^{x_{0}}}^{\prime}$. By analogy with Physics, we call this process a Lagrangian Acquisition.

Example 1.3. In the last example which illustrates the previous one, we consider an Eulerian velocity vector field $U_{t, x}$ for a fluid medium. Let $\mathcal{D}$ be a domain of the configuration space $E$, we define for all $t \in[0, T]$, the Eulerian velocity field $U_{t, x}$ as a solution of the Navier-Stokes Equation:

$$
\begin{cases}\left(\frac{\partial}{\partial t}+U_{t, x} \nabla_{x}\right) U_{t, x}=-\nabla_{x} p_{t, x}+\mu \Delta U_{t, x} & x \in \mathcal{D} \\ U_{t, x}=f(t, x) & x \text { on } \partial \mathcal{D} \\ U_{0, x}=U_{0}(x) & \end{cases}
$$

where $f$ is a well-defined function for each time $t$. With the point of departure $x_{0} \in \mathcal{D}$, the location of a fluid element is given by a solution of the Stochastic Partial Differential Equation:

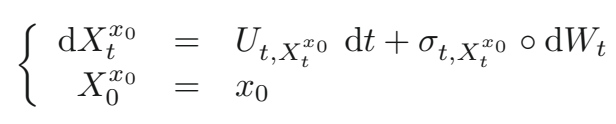

where $\left(W_{t}\right)_{t \geq 0}$ is a cylindrical Brownian Motion and $\sigma_{t, x}$ a known nonzero function. With the regularity hypothesis given by [14], Mikulevicius and Rozovskii claim the existence of mild solutions for the two systems (in small time). The pair $\left(X_{t}, U_{t, x}\right)$ is an $\left((E, \mathcal{E}) \times\left(E^{\prime}, \mathcal{E}^{\prime}\right)\right.$ )-valued Acquisition System. We define the Acquisition Process by $A_{t}=U_{t, X_{t}^{x_{0}}}$. Then $A_{t}$ is the Lagrangian velocity usually denoted $V_{t}$ of a fluid element started at $x_{0}$ and carried out by the flow $X_{t}^{x_{0}}$.

With these first three examples, we can notice that the stochastic process $A_{t}$ located $X_{t}$ do not require the knowledge of the vector field everywhere. More, the nature of $A_{t}$ is utterly linked to the process $X_{t}$. Thus, the probabilistic properties of the field (Markovian, ergodicity, etc.) will not be given only relatively to the random variable $A_{t}$, but more precisely relatively to the pair $\left(X_{t}, A_{t}\right)$.

For all $t \in[0, T]$, we suppose the absolute continuity of the law of the pair $\left(X_{t}, A_{t}\right)$ w.r.t. Lebesgue measure with the density $p^{X_{t}, A_{t}}$. We assume that for any $x \in E, \int_{E^{\prime}} p^{X_{t}, A_{t}}(x, a) \mathrm{d} a>0$ and the existence of the conditional density $p^{A_{t} \mid X_{t}}$. By the definition of the conditional expectation knowing $X_{t}$ for all bounded measurable functions $f$ from $\left(E, E^{\prime}\right)$ to $\mathbb{R}$ :

$$
\mathbb{E}\left(f\left(X_{t}, A_{t}\right) \mid X_{t}\right)=\int_{E^{\prime}} f\left(X_{t}, a\right) p^{A_{t} \mid X_{t}}\left(a \mid X_{t}\right) \mathrm{d} a
$$

the conditional law is given by the joint pdf:

$$
p^{A_{t} \mid X_{t}}(a \mid x)=\frac{p^{X_{t}, A_{t}}(x, a)}{\int_{E^{\prime}} p^{X_{t}, A_{t}}(x, a) \mathrm{d} a} .
$$

In physical experiments or in engineering applications, it could be necessary to evaluate only the expectation for $A_{t}$ instead of for the pair $\left(X_{t}, A_{t}\right)$. It is the case of measurements on a random medium where no information of the geometry of the medium is available (fluid flow, small configuration of a nuclear reactor, etc.). 
Then the classic method requires to use a weak regularizing $\mathcal{C}^{\infty}$ kernel $K^{\delta}$ of the Dirac measure, $\delta$ being a cut-off parameter. Then for all $t \in[0, T]$, for any measurable bounded function $f$, the conditional expectation of $A_{t}$ given $X_{t}$ is approximated by:

$$
\mathbb{E}^{\delta}\left(f\left(A_{t}\right) \mid X_{t}\right)=\int_{E \times E^{\prime}} f(a) K^{\delta}\left(X_{t}, z\right) p^{A_{t} \mid X_{t}}(a \mid z) \mathrm{d} a \mathrm{~d} z .
$$

More exactly, using the joint law $p^{X_{t}, A_{t}}$, we define the regularized integral:

$$
\mathbb{F}^{\delta}\left(f\left(A_{t}\right)\right)\left(X_{t}\right)=\int_{E \times E^{\prime}} f(a) K^{\delta}\left(X_{t}, z\right) p^{X_{t}, A_{t}}(z, a) \mathrm{d} z \mathrm{~d} a .
$$

We assume here that $\int_{E \times E^{\prime}} K^{\delta}\left(X_{t}, z\right) p^{X_{t}, A_{t}}(z, a) \mathrm{d} z \mathrm{~d} a$ is not null. Then the regularized expectation can be expressed in terms of $\mathbb{F}^{\delta}$ :

$$
\mathbb{E}^{\delta}\left(f\left(A_{t}\right) \mid X_{t}\right)=\frac{\mathbb{F}^{\delta}\left(f\left(A_{t}\right)\right)\left(X_{t}\right)}{\mathbb{F}^{\delta}(1)\left(X_{t}\right)}
$$

with some necessary regularity properties on the density given by a supplementary hypothesis, it holds

$$
\lim _{\delta \rightarrow 0} \mathbb{E}^{\delta}\left(f\left(A_{t}\right) \mid X_{t}\right)=\mathbb{E}\left(f\left(A_{t}\right) \mid X_{t}\right) .
$$

Now, here come two applications directly connected to our previous examples.

Application 1.1. This is the trivial case where for a fixed point $x \in E$, for all time $t>0$, we have the stationary Acquisition Path $X_{t}=x$. We define the Acquisition Field as the Eulerian velocities vector field $U_{t, x}$ given by the NSE (1.1). Then $U_{t}=U_{t, x}$ is an Acquisition Process and for all bounded measurable function $f$, we have $\mathbb{E}\left(f\left(U_{t, X_{t}}\right) \mid X_{t}=x\right) \stackrel{p . s .}{=} \mathbb{E}\left(f\left(U_{t}\right)\right)$. This is the usual Eulerian velocities mean usually written in fluid mechanics literature (with a chaotic point of view) by the Ensemble Average $\langle f(U)\rangle_{t, x}$. In the sequel we will use this remark and replace for a fluid, the ensemble average $\langle f(U)\rangle_{t, x}$ by the conditional expectation $\mathbb{E}\left(f\left(U_{t, X_{t}}\right) \mid X_{t}=x\right)$.

Application 1.2. Another direct application, to stay in the fluid mechanics domain, let $U_{t, x}$ be the Acquisition Field given by the Eulerian velocities vector field. Let $X_{t}^{x_{0}}$ be the Acquisition Path defined by the trajectory associated to the field with the point of departure $x_{0} \in E$. This trajectory is given by the solution of the equation (1.2).

The Lagrangian Acquisition is defined by $V_{t}=U_{t, X_{t}^{x_{0}}}$ and using the regularised expectation we have

$$
\mathbb{E}\left(f\left(V_{t}\right) \mid X_{t}\right)=\lim _{\delta \rightarrow 0} \frac{\mathbb{F}^{\delta}\left(f\left(V_{t}\right)\right)\left(X_{t}\right)}{\mathbb{F}^{\delta}(1)\left(X_{t}\right)}
$$

with $\mathbb{F}^{\delta}\left(f\left(V_{t}\right)\right)\left(X_{t}\right)=\int_{E \times E^{\prime}} f(v) K^{\delta}\left(X_{t}, x\right) p^{X_{t}, V_{t}}(x, v) \mathrm{d} x \mathrm{~d} v$.

For example, in the case of homogeneous turbulent flows using the Kraichnan model of turbulence, the regularizing kernel can be given by the Gaussian function:

$$
K^{\delta}\left(X_{t}, x\right)=G^{\delta}\left(X_{t}-x\right)=\mathrm{e}^{\frac{-\left\|X_{t}-x\right\|^{2}}{2 \delta}} .
$$

Pope in [15] proposes other kernels built using spline functions and phenomenological considerations.

Remark 1.1. A filtering problem can be considered as the symmetrical problem of the Acquisition estimation. Indeed an observation $Y_{t} \in E^{\prime}$ of a state $\mathcal{X}_{t} \in E$ are connected together by the observational equation $Y_{t}=H\left(t, \mathcal{X}_{t}, W_{t}\right)$ where $W_{t}$ is a canonical random process, and $H$ is a bounded transfer function. Clearly the transfer function $H\left(t, \mathcal{X}_{t}, \cdot\right) \stackrel{\text { def }}{=} H_{t, \mathcal{X}_{t}}(\cdot)$ plays the role of a random field and $Y_{t}$ is the Acquisition Process 
of the acquisition field $H$ along the path $\mathcal{X}_{t}$ in $E$. The acquisition problem consists in the computation of $\mathbb{E}\left(f\left(Y_{t}\right) \mid \mathcal{X}_{t}\right)$ while the filtering problem is the calculation of $\mathbb{E}\left(f\left(\mathcal{X}_{t}\right) \mid Y_{t}\right)$. This remark is fruitful to suggest an algorithm estimating the Acquisition expectations.

\section{ESTIMATION FOR DISCRETE ACQUISITION IN LOCALLY HOMOGENEOUS MEDIUM}

As said previously the estimation of the medium parameter along a path is a pointwise process and we have to derive the dynamics of the mobile measurements from a local model. In this section, we propose a sequential algorithm for the computation of the Acquisition Process expectation given the Acquisition Path. We consider an Acquisition System $\left(X, X^{\prime}\right)$ with a discrete time dynamics. Let be $n \in \mathbb{N}, \Delta t>0$ a time step, and for all $i \in\{0, \ldots, n\}$ we denote $t_{i}=i \Delta t$ and $\left(X_{i}, X_{i, x}^{\prime}\right) \stackrel{\text { def }}{=}\left(X_{t_{i}}, X_{t_{i}, x}^{\prime}\right)$.

Definition 2.1 (Locally homogeneous Acquisition Process). For all $n \in \mathbb{N}$, let $\left(X_{n}, X_{n, x}^{\prime}\right)$ be an $\left(E \times E^{\prime}\right)$ valued Acquisition System and $A_{n}=X_{n, X_{n}}^{\prime}$ an Acquisition Process. The Acquisition System is said to be locally homogeneous if:

- $E$ is a metrisable locally convex space with a convex set covering $\mathcal{A}=\bigcup_{i \in I} \mathcal{A}_{i}$, where $I$ is an index set.

- $\forall n \in \mathbb{N}$ and $\forall x \in E$, there exists $\varepsilon_{n}>0$ and $i \in I$ such that:

$B\left(x, \varepsilon_{n}\right) \subset \mathcal{A}_{i}$ with $B\left(x, \varepsilon_{n}\right)=\left\{z \in E\right.$ s.t. $\left.|x-z| \leq \varepsilon_{n}\right\}$ and $\forall y \in B\left(x, \varepsilon_{n}\right)=B_{n}^{\varepsilon}(x)$, and for all $a \in E^{\prime}$ we have $\mathbb{P}\left(A_{n} \in d a \mid X_{n}=x\right)=\mathbb{P}\left(A_{n} \in d a \mid X_{n}=y\right)$.

This is the law of $A_{n}$ given $X_{n}$ belonging to the ball $B_{n}^{\varepsilon}(x)$, denoted Law $\left(A_{n} \mid X_{n} \in B_{n}^{\varepsilon}(x)\right)$.

In the locally homogeneous case, it will be possible to evaluate the expectation $\mathbb{E}\left(f\left(X_{n}, A_{n}\right) \mid X_{n} \in B_{n}^{\varepsilon}\right)$. First of all, we highlight that:

$$
\begin{aligned}
\mathbb{E}\left(f\left(X_{n}, A_{n}\right) \mid X_{n} \in B_{n}^{\varepsilon}\right) & =\int_{E \times E^{\prime}} f(x, a) p^{A_{n} \mid X_{n} \in B_{n}^{\varepsilon}}(a \mid x) \mathrm{d} x \mathrm{~d} a=\int_{E \times E^{\prime}} f(x, a) \frac{\mathbb{1}_{B_{n}^{\varepsilon}}(x) p^{X_{n}, A_{n}}(x, a)}{\int_{E} \mathbb{1}_{B_{n}^{\varepsilon}}(x) p^{X_{n}}(x) \mathrm{d} x} \mathrm{~d} x \mathrm{~d} a \\
& =\frac{\mathbb{E}\left(f\left(X_{n}, A_{n}\right) \mathbb{1}_{B_{n}^{\varepsilon}}\left(X_{n}\right)\right)}{\mathbb{E}\left(\mathbb{1}_{B_{n}^{\varepsilon}}\left(X_{n}\right)\right)} .
\end{aligned}
$$

As described in Figure 1, this definition is directly extendable to the trajectorial processes. For $n>0$, we denote $X_{[0, n]}=\left\{X_{i}, i \in\{0, \ldots, n\}\right\}$ the discrete path of the process $X_{n}$. Let $B_{[0, n]}$ be the hull of the balls set $\left(B_{i}^{\varepsilon}\right)_{i \leq n}$ :

$$
B_{[0, n]}=\bigcup_{i \in\{0, \ldots, n\}} B_{i}^{\varepsilon}\left(x_{i}\right) .
$$

We denote $X_{[0, n]} \in B_{[0, n]}$ the event $\left\{X_{0} \in B_{0}^{\varepsilon}\left(x_{0}\right), \ldots, X_{n} \in B_{n}^{\varepsilon}\left(x_{n}\right)\right\}$.

In this trajectorial situation we attempt to the computation of the probability measures $\chi_{n}$ such that for any bounded measurable function $f$ we get the acquisition expectation along the path $X_{[0, n]}$ :

$$
\chi_{n}(f)=\mathbb{E}\left(f\left(X_{n}, A_{n}\right) \mid X_{[0, n]} \in B_{[0, n]}\right) .
$$

The structure of the conditional expectation $\chi_{n}$ suggests with its product of (indicatrice) functions a possible Feynman-Kac shape (see [9] for a complete description of Feynman-Kac measures).

\subsection{Mean-field interpretation for the discrete acquisition process}

Up to now, we have introduced the Acquisition Systems in their general form, with examples coming from fluid mechanics. To go further, we have to specialize the description mainly taking into account a local dynamical structure. General Acquisition Processes indeed have structures close the ones occur in a filtering problem. 


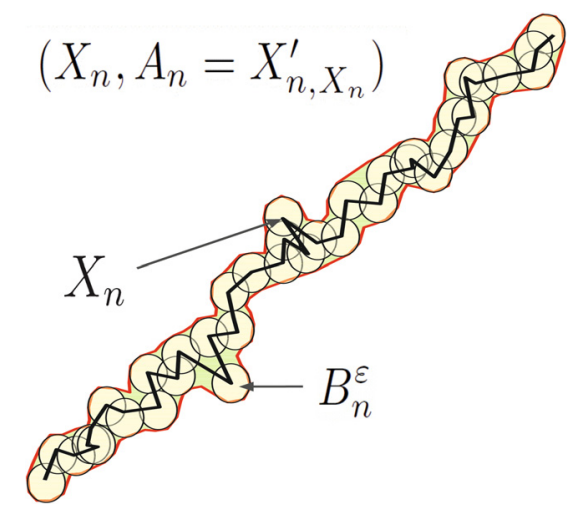

Figure 1. Set of homogeneity balls for a Acquisition Path.

There is an a-priori evolution of the medium (i.e. without interaction with the Acquisition Path) and then a conditioning to the Acquisition Field statement along the Acquisition Path. This procedure can be decomposed in two steps, one for the medium's prediction stage and the second for a selection along the Acquisition Path.

Since the medium is only locally known by the Acquisition, the model can be local and we choose to use a probabilistic representation with a Lagrangian dynamics. We have also seen that a Lagrangian dynamics is a specific Acquisition Process and we realize the coupling between this Lagrangian Acquisition and the Acquisition Process associated with the random path of measurements.

For a path initiated at $x_{0} \in E$, we denote $X_{i}^{\prime}=X_{i, X_{i}^{x_{0}}}^{\prime}$ the discrete Lagrangian Acquisition. Let $Z_{0}, \ldots, Z_{n}$ be a discrete path in the configuration space $E$ independent of the Lagrangian Acquisition dynamics.

In the situation of a locally homogeneous medium, the knowledge of the Acquisition Field can be restricted to a ball $B_{n}^{\varepsilon}$ excluding the values of the field outside this ball.

Then to achieve the estimation we have to couple two Acquisition Systems:

- The first one is given by the Lagrangian model (1 point model) and put out the Lagrangian Acquisition with the point of departure $x_{0} \in E:\left(X_{n}^{x_{0}}, X_{n, X_{n}^{x_{0}}}^{\prime}\right)=\left(X_{n}, X_{n}^{\prime}\right)$.

- The second Acquisition system is the statement of the Lagrangian Acquisition along the independent Acquisition Path $Z_{n}:\left(Z_{n}, A_{n}\right)$.

To express what $A_{n}$ could be, we have to consider a set of Lagrangian Acquisition starting from different initial points and arriving to the different points of the Acquisition Path. Thus, we have the explanation of Figure 2:

- At the step $n-1$, the Acquisition Path is at $Z_{n-1}$, and acquires the value $X_{n-1}^{\prime}$ in the phase space given by the Lagrangian Acquisition $X_{n-1}^{x_{0}^{n-1}}=Z_{n-1}$ started from $x_{0}^{n-1}$ and then $A_{n-1}^{x_{0}^{n-1}}=X_{n-1, Z_{n-1}}^{\prime}$. The superscript $x_{0}^{n-1}$ is to emphasize the departure point of the Lagrangian Acquisition.

- At time $n$ we change of Lagrangian Acquisition to acquire those started from the initial point $x_{0}^{n}$ and arrived at $Z_{n}$. Then we got $A_{n}^{x_{0}^{n}}=X_{n, Z_{n}}^{\prime}$.

But we do not have any informations of the point of departure for each Lagrangian trajectory. It would be suitable to compute the expectation $A_{n}=\int A_{n}^{x} \mathbb{P}^{X_{0}}(\mathrm{~d} x)$. This expectation is hard to approach, it is an integration along the ancestral lines.

Therefore, using the locally homogeneous medium hypothesis, we propose to give in each point of the Acquisition Path $Z_{n}$ a parameter $\varepsilon_{n}>0$ and a ball $B_{n}^{\varepsilon}\left(Z_{n}\right)=\left\{x \in E:\left|x-Z_{n}\right| \leq \varepsilon_{n}\right\}$. Then we estimate 


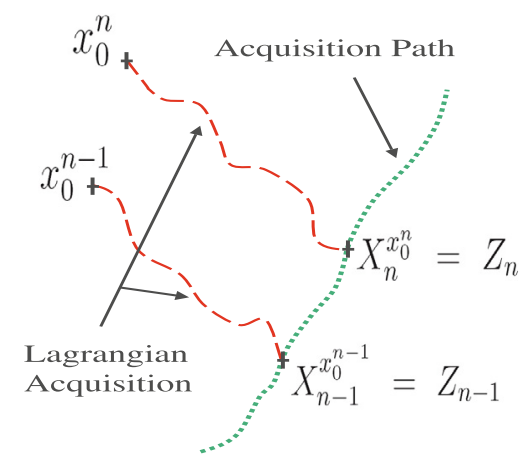

Figure 2. Coupling between a Lagrangian Acquisition and an Acquisition along an independent random path.

the two probability measures $\chi_{n}$ and $\hat{\chi}_{n}$ such that for any test function $f$ :

$$
\begin{aligned}
& \hat{\chi}_{n}(f)=\mathbb{E}\left(f\left(X_{n}, A_{n}\right) \mid X_{0} \in B_{0}^{\varepsilon}\left(Z_{0}\right), \ldots, X_{n} \in B_{n}^{\varepsilon}\left(Z_{n}\right)\right) \\
& \chi_{n}(f)=\mathbb{E}\left(f\left(X_{n}, A_{n}\right) \mid X_{0} \in B_{0}^{\varepsilon}\left(Z_{0}\right), \ldots, X_{n-1} \in B_{n-1}^{\varepsilon}\left(Z_{n-1}\right)\right) .
\end{aligned}
$$

Complementary with the Lagrangian hypothesis, the pair $\left(X_{n}, X_{n}^{\prime}\right)$ is supposed to be Markovian and the evolution is described by the mean-field transition kernel:

$$
M_{n+1, \pi_{n}}\left(\left(x, x^{\prime}\right), d\left(z, z^{\prime}\right)\right)=\mathbb{P}\left(\left(X_{n+1}, X_{n+1}^{\prime}\right) \in d\left(z, z^{\prime}\right) \mid\left(X_{n}, X_{n}^{\prime}\right)=\left(x, x^{\prime}\right)\right)
$$

where $\pi_{n}$ is the probability law of $\left(X_{n}, X_{n}^{\prime}\right)$. Later we will call the evolution using the transition kernel $M_{n+1, \pi_{n}}$ a mutation. The mean-field hypothesis is made to take into account the macroscopic structures of the random field locally represented by $\left(X_{n}, X_{n}^{\prime}\right)$.

Being inspired by (2.1), we can write:

$$
\begin{aligned}
\hat{\chi}_{n}(f) & =\frac{\mathbb{E}\left(f\left(X_{n}, X_{n}^{\prime}\right) \prod_{p=0}^{n} \mathbb{1}_{B_{p}^{\varepsilon}\left(Z_{p}\right)}\left(X_{p}\right)\right)}{\mathbb{E}\left(\prod_{p=0}^{n} \mathbb{1}_{B_{p}^{\varepsilon}\left(Z_{p}\right)}\left(X_{p}\right)\right)} \\
\chi_{n}(f) & =\frac{\mathbb{E}\left(f\left(X_{n}, X_{n}^{\prime}\right) \prod_{p=0}^{n-1} \mathbb{1}_{B_{p}^{\varepsilon}\left(Z_{p}\right)}\left(X_{p}\right)\right)}{\mathbb{E}\left(\prod_{p=0}^{n-1} \mathbb{1}_{B_{p}^{\varepsilon}\left(Z_{p}\right)}\left(X_{p}\right)\right)}
\end{aligned}
$$

which confer on the probability measures $\hat{\chi}_{n}$ and $\chi_{n}$ their Feynman-Kac distribution structure.

Without any change we do not only consider the conditioning given the ball $\mathbb{1}_{B_{p}^{\varepsilon}\left(Z_{p}\right)}$ but given the cylinder $\mathbb{1}_{B_{p}^{\varepsilon}\left(Z_{p}\right) \times E^{\prime}}$ and we define the potential $G_{p}$ for $\left(X_{p}, X_{p}^{\prime}\right)$ :

$$
G_{p}\left(\left(X_{p}, X_{p}^{\prime}\right)\right)=\mathbb{1}_{B_{p}^{\varepsilon}\left(Z_{p}\right) \times E^{\prime}}\left(\left(X_{p}, X_{p}^{\prime}\right)\right) .
$$

Associated to this potential function, we define the selection kernel:

$$
\begin{aligned}
S_{n+1, \chi_{n+1}}^{Z}\left(\left(x, x^{\prime}\right), d\left(y, y^{\prime}\right)\right)=\mathbb{1}_{B_{n+1}^{\varepsilon}\left(Z_{n+1}\right) \times E^{\prime}}\left(x, x^{\prime}\right) \delta_{\left(x, x^{\prime}\right)}\left(d\left(y, y^{\prime}\right)\right) & \\
& +\left[1-\mathbb{1}_{B_{n+1}^{\varepsilon}\left(Z_{n+1}\right) \times E^{\prime}}\left(x, x^{\prime}\right)\right] \frac{\mathbb{1}_{B_{n+1}^{\varepsilon}\left(Z_{n+1}\right) \times E^{\prime}}\left(y, y^{\prime}\right) \chi_{n+1}\left(d\left(y, y^{\prime}\right)\right)}{\chi_{n+1}\left(B_{n+1}^{\varepsilon}\left(Z_{n+1}\right) \times E^{\prime}\right)}
\end{aligned}
$$

where $\chi_{n+1}\left(d\left(y, y^{\prime}\right)\right)=\mathbb{P}\left(\left(X_{n+1}, X_{n+1}^{\prime}\right) \in d\left(y, y^{\prime}\right) \mid X_{0} \in B_{0}^{\varepsilon}\left(Z_{0}\right), \ldots, X_{n} \in B_{n}^{\varepsilon}\left(Z_{n}\right)\right)$. 


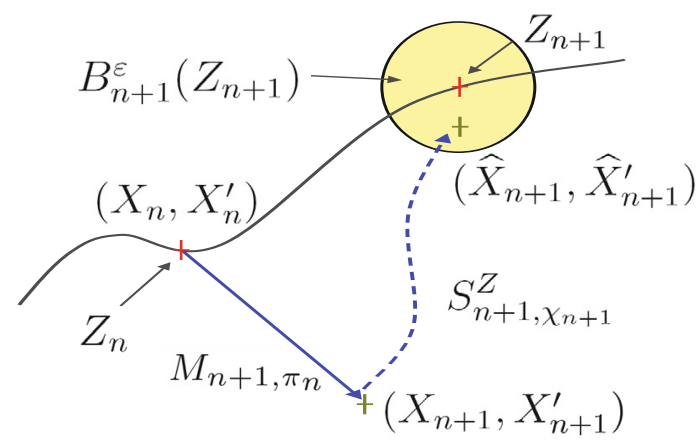

Figure 3. Discrete evolution of the Acquisition Process for a Lagrangian dynamics.

Starting from the Acquisition initial distribution

$$
\chi_{0}\left(d\left(x, x^{\prime}\right)\right)=\mathbb{P}\left(\left(X_{0}, X_{0}^{\prime}\right) \in d\left(x, x^{\prime}\right)\right)
$$

we have the sequential scheme, for $n \geq 0$ :

$$
\hat{\chi}_{n} \stackrel{M_{n+1, \pi_{n}}}{\longrightarrow} \chi_{n+1} \stackrel{S_{n+1, \chi_{n+1}}^{Z}}{\longrightarrow} \hat{\chi}_{n+1}
$$

which is a stochastic dynamical system: $\chi_{n+1}=\chi_{n} S_{n, \chi_{n}}^{Z} M_{n+1, \pi_{n}}$.

In terms of the state vector evolution, Figure 3 illustrates the procedure which corresponds to:

$$
\left(\hat{X}_{n}, \hat{X}_{n}^{\prime}\right) \stackrel{\text { Mutation }}{\longrightarrow}\left(X_{n+1}, X_{n+1}^{\prime}\right) \stackrel{\text { Selection }}{\longrightarrow}\left(\hat{X}_{n+1}, \hat{X}_{n+1}^{\prime}\right)
$$

After the selection step, the state $\left(\hat{X}_{n+1}, \hat{X}_{n+1}^{\prime}\right)$ is the estimation of the Acquisition System, where $\hat{X}_{n+1}$ is the location of the system and $\hat{X}_{n+1}^{\prime}$ is the estimation of the required Acquisition Process $A_{n+1}=X_{n+1, Z_{n+1}}^{\prime}$.

The coupling between the two Acquisition Systems is a localization after a Lagrangian mutation. Written like this, the evolution is not Markovian and the potential can nullify. The Figure 3 is quite abusive, we must have a covering of the trajectory $Z_{0}, \ldots, Z_{n}$ by balls $\left(B_{j}^{\varepsilon}\left(Z_{j}\right)\right)_{0 \leq j \leq n}$ with non-empty intersections as seen in Figure 2.

For each time step $n$ and each state point $\left(x, x^{\prime}\right)$, we suppose that $0<M_{n+1, \pi_{n}}\left(\mathbb{1}_{B_{n+1}^{\varepsilon}\left(Z_{n+1}\right) \times E^{\prime}}\right)\left(x, x^{\prime}\right)$. This hypothesis means that the probability to arrive in a ball $B_{n+1}^{\varepsilon}\left(Z_{n+1}\right)$ coming from $x$ is not null. This hypothesis is important, without we lost the Markovian nature of the process. It has a non-trivial implications. For example in physical applications, the time-discretization should be tight enough.

Now we define the modified Markov kernel and the new potential function to restore Markovian property:

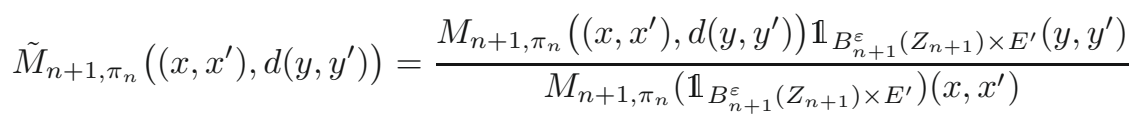

and

$$
\tilde{G}_{n+1}\left(x, x^{\prime}\right)=M_{n+1, \pi_{n}}\left(\mathbb{1}_{B_{n+1}^{\varepsilon}\left(Z_{n+1}\right) \times E^{\prime}}\right)\left(x, x^{\prime}\right) .
$$


The Markovian kernel $\tilde{M}_{n+1, \pi_{n}}\left(\left(x, x^{\prime}\right), d\left(y, y^{\prime}\right)\right)$ is a local transition restricted to the domain $B_{n+1}^{\varepsilon}\left(Z_{n+1}\right)$ while the potential function $\tilde{G}_{n+1}\left(x, x^{\prime}\right)$ gives the chance to evolve in $B_{n+1}^{\varepsilon}\left(Z_{n+1}\right) \times E^{\prime}$. This implies that the Lagrangian Acquisition follows the path $Z_{0}, \ldots, Z_{n+1}$ and the potential can not be degenerate.

We start at initial step from the cylinder $B_{0}^{\varepsilon}\left(Z_{0}\right) \times E^{\prime}$ using the probability $\chi_{0}^{B}$ and the law $\pi_{0}$ of the pair $\left(X_{0}, X_{0}^{\prime}\right)$. We use for all the time step the Feynman-Kac potential and kernel $\left(\tilde{G}_{n}, \tilde{M}_{n+1, \pi_{n}}\right)$ to produce the Feynman-Kac distribution at each time step $n$. We denote $\chi_{n}^{B}$ and $\hat{\chi}_{n}^{B}$ the stochastic flows of the process restricted to the cylinders $B_{n+1}^{\varepsilon}\left(Z_{n+1}\right) \times E^{\prime}$. This corresponds to an interacting mean-field process, solution of the nonlinear equation $\chi_{n+1}^{B}=\chi_{n}^{B} K_{n+1, \chi_{n}^{B}, \pi_{n}}^{B}$.

We know that the dynamical system $\chi_{n+1}^{B}=\chi_{n}^{B} K_{n+1, \chi_{n}^{B}, \pi_{n}}^{B}$ have a non-unique McKean representation and we choose to write:

$$
K_{n+1, \chi_{n}^{B}, \pi_{n}}^{B}=\tilde{S}_{n, \chi_{n}^{B}} \tilde{M}_{n+1, \pi_{n}} .
$$

The non-unicity is brought by the selection kernel $\tilde{S}_{n, \chi_{n}^{B}}$. Using the potential function $\tilde{G}_{n+1}$ we choose a particular form for $\tilde{S}_{n, \chi_{n}^{B}}$, called genetic selection:

$$
\tilde{S}_{n+1, \chi_{n}^{B}}\left(\left(x, x^{\prime}\right), d\left(y, y^{\prime}\right)\right)=\tilde{G}_{n+1}\left(x, x^{\prime}\right) \delta_{\left(x, x^{\prime}\right)}\left(d\left(y, y^{\prime}\right)\right)+\left[1-\tilde{G}_{n+1}\left(x, x^{\prime}\right)\right] \frac{\tilde{G}_{n+1}\left(y, y^{\prime}\right) \chi_{n}^{B}\left(d\left(y, y^{\prime}\right)\right)}{\chi_{n}^{B}\left(\tilde{G}_{n+1}\right)} .
$$

The choose of the genetic selection is the justification of the name "mutation" given to the prediction step. This dynamical system produces a sequential scheme for the evolution laws restricted to the Acquisition Path:

$$
\hat{\chi}_{n}^{B} \stackrel{\tilde{M}_{n+1, \pi_{n}}}{\longrightarrow} \chi_{n+1}^{B} \stackrel{\tilde{S}_{n+1, \chi_{n+1}^{B}}}{\longrightarrow} \hat{\chi}_{n+1}^{B}
$$

and in terms of the state space points we have the algorithm:

$$
\underbrace{\left(X_{n}^{B}, X_{n}^{\prime} B\right)}_{\in\left(B_{n}^{\varepsilon}\left(Z_{n}\right) \times E^{\prime}\right)} \stackrel{\text { Selection }}{\longrightarrow} \underbrace{\left(\hat{X}_{n}^{B}, \hat{X}_{n}^{\prime} B\right)}_{\in\left(B_{n}^{\varepsilon}\left(Z_{n}\right) \times E^{\prime}\right)} \stackrel{\text { Mutation restricted to } B_{n+1}^{\varepsilon}}{\longrightarrow} \underbrace{\left(X_{n+1}^{B}, X_{n+1}^{\prime} B\right)}_{\in\left(B_{n+1}^{\varepsilon}\left(Z_{n+1}\right) \times E^{\prime}\right)}
$$

The Acquisition flow restricted to the balls centered to the Acquisition Path is therefore a solution of the dynamical system $\chi_{n+1}^{B}=\chi_{n}^{B} \tilde{S}_{n, \chi_{n}^{B}} \tilde{M}_{n+1, \pi_{n}}$ with initial $\chi_{0}^{B}$ and describes the coupling between the two Acquisition Systems along the random path $Z_{0}, \ldots, Z_{n}$ and it is a mean-field process.

Now we have to develop a particle approximation of this Acquisition Process.

\subsection{Particle approximation of acquisition processes on locally homogeneous medium}

The Acquisition Process is given by the solution of stochastic integrals. These integrals have only approximated solutions. We describe briefly the interacting particle algorithm. The algorithm will be more detailed in the next section where the Acquisition Process will be used to filter pointwise measurements on a random medium.

We have by hypotheses a $E$-valued Acquisition Path $\left(Z_{0}, \ldots, Z_{n}\right)$ and because of the locally homogeneous medium a set of real numbers $0<\left(\varepsilon_{i}\right)_{0 \leq i \leq n}$ with the existence of homogeneity balls $B_{i}^{\varepsilon}\left(Z_{i}\right)$ for $i \in\{0, \ldots, n\}$.

To establish the asymptotic approximations of the particle system, the Markovian transition have to be sufficiently regular, at least for a class of function. So we assume that the Markovian transition $\tilde{M}_{n+1, \pi_{n}}$ follows a regularity hypothesis: For any measurable bounded function $f$ and any measure $\pi, \forall n \in \mathbb{N}$, there exists some constants $c_{n}(\pi)$ and a finite set of bounded functions $h$ with $\|h\| \leq 1$ such that for any measure $\mu$

$$
\left\|\tilde{M}_{n+1, \pi}(f)-\tilde{M}_{n+1, \mu}(f)\right\| \leq c_{n}(\pi)\|f\| \sum_{h}|\pi(h)-\mu(h)| .
$$


For the initial step, we consider a ball $B_{0}^{\varepsilon}\left(Z_{0}\right) \times E^{\prime}$ and a particle system with cardinal $N>0,\left(\xi_{0}^{i}, \xi_{0}^{\prime i}\right)_{1 \leq i \leq N}$, i.i.d. following $\chi_{0}^{B}$ and we denote:

$$
\chi_{0}^{B, N}=\frac{1}{N} \sum_{i=1}^{N} \delta_{\xi_{0}^{i}, \xi_{0}^{\prime}} .
$$

The empirical law $\chi_{0}^{B, N}$ is an approximation of the exact law $\chi_{0}^{B}$ and the technical Lemma 3.1 gives the existence for all $p>0$ of a constant $C_{0}(p)$ such that:

$$
\mathbb{E}\left(\left\|\chi_{0}^{B, N}-\chi_{0}^{B}\right\|_{\mathcal{H}}^{p}\right)^{\frac{1}{p}} \leq \frac{C_{0}(p)}{\sqrt{N}} I(\mathcal{H}) .
$$

At the beginning of the $n$th time step, we have a particle system $\left(\hat{\xi}_{n}^{i}, \hat{\xi}_{n}^{\prime}\right)_{1 \leq i \leq N}$ located in the ball $B_{n}^{\varepsilon}\left(Z_{n}\right) \times E^{\prime}$ and following the sequential scheme

$$
\left(\hat{\xi}_{n}^{i}, \hat{\xi}_{n}^{\prime}\right) \stackrel{\text { Mutation }}{\longrightarrow}\left(\xi_{n+1}^{i}, \xi_{n+1}^{\prime i}\right) \stackrel{\text { Selection }}{\longrightarrow}\left(\hat{\xi}_{n+1}^{i}, \hat{\xi}_{n+1}^{i}\right) .
$$

The exact flow $\chi_{n+1}^{B}=\chi_{n}^{B} K_{n+1, \chi_{n}^{B}, \pi_{n}}^{B}$ is approached by $\chi_{n+1}^{B, N}$ obtained by the empirical transition $K_{n+1, \chi_{n}^{B}, \pi_{n}}^{B}$ of the discrete law $\chi_{n}^{B, N}=\frac{1}{N} \sum_{i=1}^{N} \delta_{\left(\xi_{n}^{i}, \xi_{n}^{\prime i}\right)}$.

As we will see in the next section, to estimate $\pi_{n}$ the a-priori law of the random medium it is necessary to have an auxiliary system with $d$ particles evolving separately with the transition kernel $\tilde{M}_{n+1, \pi_{n}}$. Then the following theorem holds:

Theorem 2.1. For all $n \geq 0$ and for all $p \geq 1$, there exists some constants $C_{n}$ and $C_{n}^{\prime}$ such that

$$
\mathbb{E}\left(\left\|\chi_{n}^{B, N}-\chi_{n}^{B}\right\|_{\mathcal{H}}^{p}\right)^{\frac{1}{p}} \leq\left[\frac{C_{n}}{\sqrt{N}}+\frac{C_{n}^{\prime}}{\sqrt{d}}\right] I(\mathcal{H})
$$

The proof of this theorem is similar to the proof of Theorem 3.2. The definitions of the subset $\mathcal{H}$, the integral entropy $I(\mathcal{H})$ and the norm $\|.\|_{\mathcal{H}}$ are given in Section 3.

One of the difficulties of this particle estimation, where the Acquisition Path is recovered with a discrete set of balls, is the possibility to nullify the potential function, $G_{n}\left(x, x^{\prime}\right)=\mathbb{1}_{B_{n}^{\varepsilon}\left(Z_{n}\right) \times E^{\prime}}\left(x, x^{\prime}\right)$ or the possibility to fail the positivity hypothesis for $M_{n+1, \pi_{n}}\left(\mathbb{1}_{B_{n+1}^{\varepsilon}\left(Z_{n+1}\right) \times E^{\prime}}\right)\left(x, x^{\prime}\right)$. In these cases, the algorithm is stopped and the estimated state is put to a cemetery point $\mho$. The estimation of this stopping time is always an open problem. Nevertheless denoting $\tau^{N}$ the first time of extinction, for some supplementary hypothesis, it is possible to estimate:

Theorem 2.2 ([9], Thm. 7.4.1). Suppose we have, for any $n \geq 0, \mathbb{E}\left(\prod_{p=0}^{n} \mathbb{1}_{B_{p}^{\varepsilon}\left(Z_{p}\right)}\left(X_{p}\right)\right)>0$. Then for all $N \geq 1$ it yields

$$
\mathbb{P}\left(\tau^{N} \leq n\right) \leq a(n) \mathrm{e}^{-N / b(n)} .
$$

For a complete proof, see [9].

This inequality is not optimal, but could indicate that with a large enough number of particles, the extinction becomes weakly probable. This could be seen as a constraint in numerical experiments and suggests that the recovering set of ball have to be wide enough without failure of the medium homogeneity hypothesis.

\section{NON-LINEAR PARTICLE FILTER FOR ACQUiSITION PROCESSES}

We present in this section the specific filter necessary to denoise corrupted Acquisition Process on homogeneous medium. To perform this filtering, we have to develop an original particle algorithm and prove its convergence. This filtering is not only suitable to Acquisition Process but is available for all mean-field processes. 
So we use generic notations which can be applied to all situations where the dynamical processes move according to a probability law.

Considering $X_{n}$ a discrete process taking values in a Polish space $(E, \mathcal{F}, \mathbb{P})$, we denote $\pi_{n}$ the law of the process $X_{n}$. For the initial step, we suppose that $X_{0}$ is a random variable distributed with the probability $\eta_{0}$.

For any $n \geq 0$, we assume that the pair $\left(X_{n}, \pi_{n}\right)$ is Markovian with the dynamical equation:

$$
X_{n+1}=X_{n}+b\left(X_{n}, \pi_{n}\right) \Delta t+\sigma_{n}^{X} \Delta W_{n}^{X}
$$

where $\Delta W_{n}^{X}$ is a sequence of i.i.d. centered Gaussian random variables with variance $\Delta t, b$ is a locally bounded function and Lipschitz in law, $\Delta t$ is the time step and $\sigma_{n}^{X}$ is a positive bounded constant.

This equation is a discrete version of a McKean SDE widely studied by Sznitman [16] and Méléard [13], and we know, with the previous hypotheses, the pathwise existence and uniqueness of the solution. For the interacting particle systems, Sznitman [16] proved the propagation of chaos property and gave some estimations for the particle approximation errors. Bossy and Talay [5] complete these works with the convergence estimations of the discretization Euler scheme.

We choose a particular shape for the function $b$ and suppose that for any $x \in E$ and for any probability measure $\mu$ on $E$ :

$$
b(x, \mu)=D(x)+\int B(x, y) \mu(\mathrm{d} y)
$$

where $D$ is a bounded function and $B$ is a bounded function for the first variable, which means that there exists $C^{B}$ such that for all $x^{\prime} \in E,\left\|B\left(., x^{\prime}\right)\right\| \leq C^{B}$. Moreover we suppose that the function $b:\left(x^{\prime}, \mu\right) \in$ $E \times \mathcal{M}_{1}(E) \rightarrow b\left(x^{\prime}, \mu\right)$ is Lipschitz with the parameter $L^{B}$ for its second variable and for all $x \in E$ and $(\mu, \nu) \in \mathcal{M}_{1}(E) \times \mathcal{M}_{1}(E)$,

$$
\|b(x, \mu)-b(x, \nu)\| \leq L^{B}\|\mu-\nu\|_{\mathcal{H}}
$$

where $\|\cdot\|_{\mathcal{H}}$ is the seminorm for $\mathcal{H}$ the sub-class of functions defined as follows:

We consider $\mathcal{H}$ a subset of bounded measurable functions $h$ such that $\|h\| \leq 1$ completed with the unity function (see [9] for details). For two measures $\mu$ and $\nu$ in $\mathcal{M}_{1}(E)$, we denote the seminorm in $\mathcal{H}$ by:

$$
\|\mu-\nu\|_{\mathcal{H}}=\sup \{|\mu(h)-\nu(h)| \text { s.t. } h \in \mathcal{H}\} .
$$

To measure a class of $\mathcal{H}$, we count the minimal number of covering $L^{p}$-balls with radius $\varepsilon>0$. This number is denoted $\mathcal{N}(\varepsilon, \mathcal{H})$, and we defined the associated integral entropy $I(\mathcal{H})=\int_{0}^{1} \sqrt{\log (1+\mathcal{N}(\varepsilon, \mathcal{H}))} \mathrm{d} \varepsilon$.

Now we given a very useful technical lemma for convergence estimation of empirical processes.

Lemma 3.1 (technical lemma). For any $p \geq 1$, any measure $\mu$ in $\mathcal{M}_{1}(E)$ and its $N$-empirical approximation $\mu^{N}, N \in \mathbb{N}^{*}$, we have

$$
\mathbb{E}\left(\left\|\mu-\mu^{N}\right\|_{\mathcal{H}}\right)^{\frac{1}{p}} \leq c[p / 2] ! I(\mathcal{H})
$$

This technical lemma and its proof are given by Del Moral in [9].

Our interest is put on the nonlinear filtering to estimate $X_{n}$, a hidden Markov mean-field process observed by the random process $Y_{n}$. For any time step $n \geq 0$, we have the system:

$$
\left\{\begin{aligned}
X_{n+1} & =X_{n}+b\left(X_{n}, \pi_{n}\right) \Delta t+\sigma_{n}^{X} \Delta W_{n}^{X} \\
Y_{n} & =h\left(X_{n}\right)+\sigma_{n}^{Y} W_{n}^{Y} \\
X_{0} & \sim \eta_{0} \\
Y_{0} & =y_{0}
\end{aligned}\right.
$$

where $h: E \rightarrow F$ is a bounded function and we assume that $F$ is a Polish space. $W_{n}^{Y}$ is a sequence of i.i.d. centered reduce Gaussian random variables independent of $\Delta W_{n}^{X}$, and $\sigma_{n}^{Y}$ a positive bounded constant. 
We denote the two Feynman-Kac flows $\left(\eta_{n}, \hat{\eta}_{n}\right)$. For all bounded measurable test function $f$, the predictor is defined by $\eta_{n}(f)=\mathbb{E}\left[f\left(X_{0} \ldots X_{n}\right) \mid Y_{0} \ldots Y_{n-1}\right]$, and the filter flow by $\hat{\eta}_{n}(f)=\mathbb{E}\left[f\left(X_{0} \ldots X_{n}\right) \mid Y_{0} \ldots Y_{n}\right]$.

The stochastic filtering problem consists in the calculation of the $\operatorname{Law}\left(X_{0} \ldots X_{n} \mid Y_{0} \ldots Y_{n}\right)$.

When the mean-field law is unknown (unfortunately, it is the general case), we have to approach this law with a (auxiliary) particle system. Thus, for any time $n \geq 0$, we need two particle systems, the first one $\left(X^{i, N}\right)_{1 \leq i \leq N}$, $N>0$ is dedicated to the filtering of the perturbed observations $Y_{n}$ and the second system $\left(Z_{n}^{i, d}\right)_{i \leq i \leq d}, d>0$ is devoted to learn the a-priori law of the medium.

For this second system, the point of departure is given by the particles $\left(Z_{0}^{i, d}\right)_{1 \leq i \leq d}$ distributed according to $\eta_{0}=\operatorname{Law}\left(X_{0}\right)$ and we suppose that for all $n>0$, each particle $Z_{n}^{i, d}$ moves with the transition kernel $M_{n+1, \pi_{n}}$ where $\pi_{n}$ is the law corresponding to the dynamical equation $Z_{n+1}^{i, d}=Z_{n}^{i, d}+b\left(Z_{n}^{i, d}, \pi_{n}\right) \Delta t+\sigma_{n}^{X} \Delta W_{n}^{Z, i}$, where $\Delta W_{n}^{Z, i}$ is a sequence of i.i.d. centered Gaussian random variables with variance $\Delta t$ independent of $\Delta W_{n}^{X}$ and $W_{n}^{Y}$.

In fact $\pi_{n}$ is unknown and each particle uses the empirical law $\pi_{n-1}^{d}=\frac{1}{d} \sum_{i=1}^{d} \delta_{Z_{n-1}^{i, d}}$. So for $i \in\{1, \ldots, d\}$, $Z_{n}^{i, d}$ evolves according to $M_{n, \pi_{n-1}^{d}}\left(Z_{n-1}^{i, d}, \cdot\right)$.

The error to the exact law can be evaluated by:

$$
\pi_{n}-\pi_{n}^{d}=\pi_{n-1} M_{n, \pi_{n-1}}-\pi_{n-1}^{d} M_{n, \pi_{n-1}^{d}}-\left[\pi_{n}^{d}-\pi_{n-1}^{d} M_{n, \pi_{n-1}^{d}}\right] .
$$

In an usual manner, $\sqrt{d}\left[\pi_{n}^{d}-\pi_{n-1}^{d} M_{n, \pi_{n-1}^{d}}\right]$ converges weakly to 0 , function by function, or almost surely, to a Gaussian random variable. We retain only the first part of the expression and consider that $\left(\pi_{n}-\pi_{n}^{d}\right)$ is approached with $\left(\pi_{n-1} M_{n, \pi_{n-1}}-\pi_{n-1}^{d} M_{n, \pi_{n-1}^{d}}\right)$ for which the technical Lemma 3.1 gives an usable error control.

Back to the filtering problem, we assume that for any time $n \geq 0$, the dynamics/observation pair $\left(X_{n}, Y_{n}\right)$ is Markovian and follows the system (3.2). For any measurable bounded test function $f$, the filtering process moves forward according to the stochastic dynamical system:

$$
\eta_{n+1}(f)=\eta_{n} K_{n+1, \eta_{n}, \pi_{n}}(f)=\eta_{n} S_{n, \eta_{n}} M_{n+1, \pi_{n}}(f) .
$$

Now we use a set of $N$ particles to estimate the filtering laws $\eta_{n}$ and $\hat{\eta}_{n}$, and a set of $d$ particles to sample the mean-field law $\pi_{n}$.

The $d$ particles $\left(Z_{n}^{i, d}\right)_{1 \leq i \leq d}$ are in interaction through their mean-field, and follow the empirical dynamics:

$$
Z_{n+1}^{i, d}=Z_{n}^{i, d}+b\left(Z_{n}^{i, d}, \pi_{n}^{d}\right) \Delta t+\sigma_{n}^{X} \Delta W_{n}^{Z, i}
$$

These particles $Z_{n}^{i, d}$ ignore the observations and evolve without selection step.

In the particle approximation of the nonlinear filter $\eta_{n+1}^{N}(f) \sim \eta_{n}^{N} S_{n, \eta_{n}^{N}} M_{n, \pi_{n}^{d}}(f)$, the empirical filtering process, the selection kernel and the mutation transition are computed using the interaction particle set $\left(X_{n}^{i, N}\right)_{1 \leq i \leq N}$ and the dynamical model:

$$
X_{n+1}^{i, N}=X_{n}^{i, N}+b\left(X_{n}^{i, N}, \pi_{n}^{d}\right) \Delta t+\sigma_{n}^{X} \Delta W_{n}^{X, i}
$$

where $\Delta W_{n}^{X, i}$ is a sequence of i.i.d. centered Gaussian random variables with variance $\Delta t$. 
We have to control the error estimation of the law $\eta_{n+1}(f)$ by the empirical law $\eta_{n+1}^{N}(f)$ and we prove the theorem:

Theorem 3.2. With the hypotheses mentioned above, for any $n \geq 0$ and $p \geq 1$, there exists finite constants $C_{n}(p)>0$ and $C_{n}^{\prime}(p)>0$ such that

$$
\mathbb{E}\left(\left\|\eta_{n}^{N}-\eta_{n}\right\|_{\mathcal{H}}^{p}\right)^{\frac{1}{p}} \leq\left[\frac{C_{n}(p)}{\sqrt{N}}+\frac{C_{n}^{\prime}(p)}{\sqrt{d}}\right] I(\mathcal{H}) .
$$

Proof. The complete proof is in the paper of Baehr and Le Gland [2] (or in [1] for this particular case) where this case and others can be found. But we can give a sketch of the proof which use triangular inequalities and argue by induction. At the initial step, the technical Lemma 3.1 gives the inequality

$$
\mathbb{E}\left(\left\|\eta_{0}^{N}-\eta_{0}\right\|_{\mathcal{H}}^{p}\right)^{\frac{1}{p}} \leq \frac{c_{p}(0)}{\sqrt{N}} I(\mathcal{H})
$$

where $C_{0}(p)=c_{0}(p)$ and $C_{0}^{\prime}(p)=0$. We show that if the statement occurs at step $n$, then it also holds at step $n+1$. We use the following decomposition:

$$
\begin{aligned}
\eta_{n+1}^{N}(f)-\eta_{n+1}(f)= & \eta_{n+1}^{N}(f)-\eta_{n}^{N} K_{n+1, \eta_{n}^{N}, \pi_{n}^{d}}(f) \\
& +\eta_{n}^{N} K_{n+1, \eta_{n}^{N}, \pi_{n}^{d}}(f)-\eta_{n}^{N} K_{n+1, \eta_{n}, \pi_{n}}(f) \\
& +\eta_{n}^{N} K_{n+1, \eta_{n}, \pi_{n}}(f)-\eta_{n} K_{n+1, \eta_{n}, \pi_{n}}(f) .
\end{aligned}
$$

The quantity $(*)$ is a fluctuation term where we obtain the control $\mathbb{E}\left((*)^{2} \mid \eta_{n}^{N}\right) \leq \frac{C}{N}\|f\|^{2}$ for some constant $C$ independent of $N$ and $d$. The difference $(* * *)$ uses the induction hypothesis, and with the notation $Q_{n+1, \pi_{n}}(z, d y)=G_{n}(z) \cdot M_{n+1, \pi_{n}}(z, d y)$, the equalities

$$
\begin{aligned}
\eta_{n}^{N} K_{n+1, \eta_{n}^{N}, \pi_{n}}(f)- & \eta_{n} K_{n+1, \eta_{n}, \pi_{n}}(f) \\
= & \eta_{n}^{N} K_{n+1, \eta_{n}^{N}, \pi_{n}}(f)-\eta_{n}^{N} K_{n+1, \eta_{n}, \pi_{n}}(f)+\eta_{n}^{N} K_{n+1, \eta_{n}, \pi_{n}}(f)-\eta_{n} K_{n+1, \eta_{n}, \pi_{n}}(f) \\
= & {\left[1-\eta_{n}^{N}\left(G_{n}\right)\right]\left(\frac{\eta_{n}^{N}}{\eta_{n}^{N}\left(G_{n}\right)}-\frac{\eta_{n}}{\eta_{n}\left(G_{n}\right)}\right) Q_{n+1, \pi_{n}}(f)+\left(\eta_{n}^{N}-\eta_{n}\right) K_{n+1, \eta_{n}, \pi_{n}}(f) } \\
= & \frac{\left[1-\eta_{n}^{N}\left(G_{n}\right)\right]}{\eta_{n}^{N}\left(G_{n}\right)}\left(\left(\eta_{n}^{N}-\eta_{n}\right) Q_{n+1, \pi_{n}}(f)+\left(\eta_{n}^{N}-\eta_{n}\right)\left(\frac{G_{n}}{\eta_{n}\left(G_{n}\right)}\right) \eta_{n} Q_{n+1, \pi_{n}}(f)\right) \\
& +\left(\eta_{n}^{N}-\eta_{n}\right) K_{n+1, \eta_{n}, \pi_{n}}(f)
\end{aligned}
$$

which gives the straightforward $L^{p}$ estimation.

For the last term $(* *)$, the work is put on the mean-field law estimation and we have to use the Lipschitz hypothesis for the function $b$.

For any bounded measurable function $f$, the difference between the exact and empirical McKean kernel is expressed by:

$$
\eta_{n}^{N} K_{n+1, \eta_{n}^{N}, \pi_{n}^{d}}(f)-\eta_{n}^{N} K_{n+1, \eta_{n}^{N}, \pi_{n}}(f)=\int f(y) \eta_{n}^{N}(\mathrm{~d} x) S_{n, \eta_{n}^{N}}(x, d z)\left[M_{n+1, \pi_{n}^{d}}-M_{n+1, \pi_{n}}\right](z, d y) .
$$

Recalling that for two $\mathcal{M}(E)$-valued measures $\mu$ and $\nu$ and any $x \in E$,

$$
M_{n+1, \mu} f(x)-M_{n+1, \nu} f(x)=\int \mathrm{d} y f(y)\left[\mathrm{e}^{\frac{-A(x, y, \mu)^{2}}{2 \sigma_{n}^{X} \Delta t}}-\mathrm{e}^{\frac{-A(x, y, \nu)^{2}}{2 \sigma_{n}^{X} \Delta t}}\right]
$$


with $A(x, y, \mu)=y-x-b(x, \mu) \Delta t$ and using the Lipschitz hypothesis plus the Gaussian properties, it yields for any $p>0$

$$
\left(\mathbb{E}\left\|\eta_{n}^{N} K_{n+1, \eta_{n}^{N}, \pi_{n}^{d}}(f)-\eta_{n}^{N} K_{n+1, \eta_{n}^{N}, \pi_{n}}(f)\right\|^{p}\right)^{\frac{1}{p}} \leq C_{n}^{\prime}(p) \frac{1}{\sqrt{d}} I(\mathcal{H}) .
$$

Using the induction, the technical Lemma 3.1 and the Marcinkiewicz' inequality, the sketch of the proof is therefore completed.

The filtering of an a-priori mean-field process requires two particle systems, one with cardinality $N$, the other with cardinality $d$, to produce bounding errors $\mathcal{O}\left(\frac{1}{\sqrt{N}}+\frac{1}{\sqrt{d}}\right)$.

Now we can modify the problem and consider a dynamical system conditioned to the observation $Y_{n}$, or more exactly to the law of the filter $\hat{\eta}_{n}$ and following the SDE:

$$
\left\{\begin{aligned}
X_{n+1} & =X_{n}+b\left(X_{n}, \hat{\eta}_{n}\right) \Delta t+\sigma_{n}^{X} \Delta W_{n}^{X} \\
Y_{n} & =h\left(X_{n}\right)+\sigma_{n}^{Y} W_{n}^{Y} \\
X_{0} & \sim \eta_{0} \\
Y_{0} & =y_{0}
\end{aligned}\right.
$$

where $\hat{\eta}_{n}$ is the $\operatorname{Law}\left(X_{n} \mid Y_{0} \ldots Y_{n}\right)$.

The filtering of this kind of process needs only one particle systems with $N$ interacting particles, and we prove the theorem:

Theorem 3.3. With the hypotheses of this section, for all $n \geq 0$ and for all $p>0$ there exists constants $0 \leq C_{n}(p)<\infty$ such that

$$
\mathbb{E}\left(\left\|\eta_{n}^{N}-\eta_{n}\right\|_{\mathcal{H}}^{p}\right)^{\frac{1}{p}} \leq \frac{C_{n}(p)}{\sqrt{N}} I(\mathcal{H})
$$

Proof. We argue by induction with analogous arguments to the previous proof. For $n=0$ invoking the technical lemma, the statement holds.

We assume that it holds for $n>0$ and for all $p>0$. For any measurable bounded function $f$, the difference $\eta_{n+1}^{N}(f)-\eta_{n+1}(f)$ can be decomposed in:

$$
\begin{aligned}
& \eta_{n+1}^{N}(f)-\eta_{n+1}(f)=\eta_{n+1}^{N}(f)-\eta_{n}^{N} K_{n+1, \eta_{n}^{N}, \hat{\eta}_{n}^{N}}(f) \\
& +\eta_{n}^{N} K_{n+1, \eta_{n}^{N}, \hat{\eta}_{n}^{N}}(f)-\eta_{n}^{N} K_{n+1, \eta_{n}^{N}, \hat{\eta}_{n}}(f) \\
& +\eta_{n}^{N} K_{n+1, \eta_{n}^{N}, \hat{\eta}_{n}}(f)-\eta_{n} K_{n+1, \eta_{n}, \hat{\eta}_{n}}(f) . \quad(* * *)
\end{aligned}
$$

Terms $(*)$ and $(* * *)$ are already known. To be controlled the term $(* *)$ uses the Lipschitz hypothesis of the function $b$ and a supplementary inequality gives the induction.

Indeed by construction, we have $\hat{\eta}_{n}=\eta_{n} S_{n, \eta_{n}}$, then the bounding verifies the inequality

$$
\left|\eta_{n} S_{n, \eta_{n}}(f)-\eta_{n}^{N} S_{n, \eta_{n}^{N}}(f)\right| \leq\left|\eta_{n} S_{n, \eta_{n}}(f)-\eta_{n} S_{n, \eta_{n}^{N}}(f)\right|+\left|\eta_{n} S_{n, \eta_{n}^{N}}(f)-\eta_{n}^{N} S_{n, \eta_{n}^{N}}(f)\right| .
$$

With some calculations on the selection kernel we have the last bound:

$$
\sup _{\|f\| \leq 1} \mathbb{E}\left|\eta_{n} S_{n, \eta_{n}}(f)-\eta_{n} S_{n, \eta_{n}^{N}}(f)\right| \leq 2 \frac{1-\eta_{n}\left(G_{n}\right)}{\eta_{n}\left(G_{n}\right)}\left\|G_{n}\right\| \sup _{\|f\| \leq 1} \mathbb{E}\left|\eta_{n}(f)-\eta_{n}^{N}(f)\right| .
$$

We use the recurrence hypothesis and obtain the required $L^{p}$ inequality for $n+1$.

In this second case, with a modified dynamical equation, with only $N$ particles we have Feynman-Kac distributions errors bounded by $\mathcal{O}\left(\frac{1}{\sqrt{N}}\right)$. This remark could be a clue to reduce the numerical cost of a filter 
for physical systems with mean-field dynamics. Accepting an error in the model we have a faster algorithm. But in counterpart, the filtering estimation is very dependent on a good representation of the observations by the used observation equation, otherwise the filter could experience serious disturbances.

\section{Application to atmospheric turbulence measurements}

In the previous two sections, we have developed a tool to inspect locally a random field, and a filtering algorithm to denoise the sort of processes implied by these local representations.

Now we propose to apply our methods to a physical problem. We consider the measurements of turbulent fluids with a special focus on observed atmospheric wind.

First, we have to choose local stochastic Lagrangian models for turbulent fluids. It will be necessary to adapt them to the measurement problem. For the adapted models, we will be able to couple the Acquisition Process dynamics and the nonlinear filtering for mean-field processes to write a performing algorithm.

We suggest here a method of closure for the dynamical system by conditioning the evolution (kernel) to the observation. With our particle approximation we will see that we have the opportunity to learn the missing parameters with the large scale observations dynamics.

For our applications, we intend to simulate data using the chosen model and the acquisition process or to use recorded real data chosen for their quality with the minimum of instrument's noises, for not to say without noise at all. These data will be called reference signals. Then, we add numerical noise with various possible type of probability laws to obtain the perturbed signal. Using this noisy signal our algorithms have to estimate the parameters of the random medium and estimate a filtered signal. As a result we can compare our estimation to the reference data which is one realization of the random medium along the path.

\subsection{Adapted stochastic Lagrangian models for nonlinear filter}

This section is devoted to the adaptation of two models for turbulent fluids. The first one is the Simplified Langevin Model (SLM) for incompressible homogeneous turbulence proposed by Pope (see [15] for example). The second model is proposed by Das and Durbin (see [8]) for relative dispersion in stratified turbulent flows.

In our filtering problem, there are no other informations on the fluid flows except the observations themselves. Thus we have to use these observations and their dynamics to learn the missing parameters. The filtering of such turbulent flows needs to constrain the model to the observations in order to give relevant estimation of the Eulerian quantities of large eddies. This conditioning to the observations of a Markovian kernel is a new method of closure for a stochastic model. Now, we detail the different necessary adaptations.

Consider for all $t \in[0, T]$ with $T \leq \infty$, the $\left(E \times E^{\prime}, \mathcal{E} \times \mathcal{E}^{\prime}\right)$-valued Acquisition System $\left(X_{t}, U_{t, x}\right)$ on $(\Omega, \mathcal{F}, \mathbb{P})$. Then vector field $U_{t, x}$ is the $(E, \mathcal{E})$-valued Eulerian fluid velocities.

The Eulerian average is given for the stationary Acquisition Path $X_{t}=x \in E$ and for all bounded measurable function $f$ by the equality $\langle f(U)\rangle_{t, x}=\mathbb{E}\left[f\left(U_{t, x}\right)\right] \mathbb{P}-p s$ (see Sect. 1, Application 1.1).

Let $x_{0} \in E$ be an initial fixed point. We consider the Lagrangian Acquisition System $\left(X_{t}^{x_{0}}, U_{t, X_{t}^{x_{0}}}=V_{t}\right)$ where $X_{t}^{x_{0}}$ is the flow coming from $x_{0}$. For any measurable function $f$ we have

$$
\mathbb{E}_{x_{0}}\left(f\left(V_{t}\right) \mid X_{t}^{x_{0}}\right) \underset{\delta \rightarrow 0}{=} \frac{\mathbb{F}_{x_{0}}^{\delta}\left(f\left(V_{t}\right)\right)}{\mathbb{F}_{x_{0}}^{\delta}(1)}\left(X_{t}^{x_{0}}\right)
$$

using the weakly regularizing kernel $G^{\delta}$ with parameter $\delta \geq 0$ mentioned in section as (1.3):

$$
\mathbb{F}_{x_{0}}^{\delta}\left(f\left(V_{t}\right)\right)\left(X_{t}^{x_{0}}\right)=\int f(v) G^{\delta}\left(X_{t}^{x_{0}}, z\right) p^{X_{t}^{x_{0}}, V_{t}}(z, v) \mathrm{d} z \mathrm{~d} v
$$

with a convergence of the approximation (4.1) as $\delta \rightarrow 0$ only for smooth densities $p^{X_{t}^{x_{0}}, V_{t}}(z, v)$. 
This expectation is dependent on the initial point $x_{0}$. If we assume that the transportation by the Lagrangian flow is without density variation (no loss or gain of mass), we can take as point of departure a random variable $X_{0}$ with the distribution $\pi_{0}$ and all the Acquisition Processes arrived at time $t$ in $x \in E$, and we evaluate the expectation $\mathbb{E}\left[f\left(V_{t}\right) \mid X_{t}=x, X_{0}\right]$ and with the same regularization technique for any measurable bounded function $f$

$$
\Pi_{t}^{\delta}\left(f\left(V_{t}\right)\right)(x) \stackrel{\text { def }}{=} \mathbb{E}^{\delta}\left[f\left(V_{t}\right) \mid X_{t}=x, X_{0}\right]
$$

and without mass density variations, we have $\mathbb{P}-p s$, for smooth densities $p^{X_{t}^{x_{0}}, V_{t}}$ :

$$
\mathbb{E}^{\delta}\left[f\left(V_{t}\right) \mid X_{t}=x\right]=\lim _{\delta \rightarrow 0} \Pi_{t}^{\delta}\left(f\left(V_{t}\right)\right)(x)
$$

This approximation $\Pi_{t}^{\delta}\left(f\left(V_{t}\right)\right)(x)$ will be used to replace the (unreachable) Eulerian average $\langle f(U)\rangle_{t, x}$.

In its continuous version for 1D or 2D isotropic flows, the simplified SLM given by Pope for incompressible isotropic homogeneous turbulence is the Itô process

$$
\left\{\begin{aligned}
\mathrm{d} X_{t} & =V_{t} \mathrm{~d} t \\
\mathrm{~d} V_{t} & =-\nabla_{x}\langle p\rangle \mathrm{d} t-\left(\frac{1}{2}+\frac{3}{4} C_{0}\right) \frac{\varepsilon_{t}}{k_{t}}\left(V_{t}-\langle v\rangle\right) \mathrm{d} t+\sqrt{C_{0} \varepsilon_{t}} \mathrm{~d} B_{t}
\end{aligned}\right.
$$

where $V_{t}$ is the Lagrangian velocity, $\nabla_{x}\langle p\rangle$ is the gradient of mean pressure, $\varepsilon_{t}$ is the turbulent dissipation rate, $k_{t}$ the Eulerian average of the turbulent kinetic energy, $\langle\cdot\rangle$ are Eulerian means, $C_{0}$ is the Kolmogorov constant and $B_{t}$ is a Wiener process. The incompressibility of the fluid requires an hypothesis: the divergence of the velocity field have to be null. This hypothesis is guaranteed by the pressure term $-\nabla_{x}\langle p\rangle \mathrm{d} t$ (see [15]).

As usually for Itô processes, the time discretization uses a classical explicit Euler scheme, here with a time step $\Delta t>0$.

In the Pope model, the quantities $-\nabla_{x}\langle p\rangle$ and $\varepsilon_{t}$ act as exogenous parameters. These two commands of the model realize a micro-macro coupling for the Lagrangian system with the large Eulerian scale.

First we express a remark due to the particular shape of equation (4.2): the asymptotic behaviour of the expectation of the velocities increments as the parameter $\delta$ tends to 0 is

$$
\mathbb{E}\left[V_{n}-\Pi_{n}^{\delta}\left(V_{n}\right)\left(X_{n}\right)\right] \stackrel{\delta \rightarrow 0}{\longrightarrow} 0
$$

Since $\nabla_{x}\langle p\rangle_{n}$ is independent of the pair $\left(X_{n}, V_{n}\right)$, it holds:

$$
\mathbb{E}\left[\Delta V_{n} \mid X_{n}\right]=-\nabla_{x}\langle p\rangle_{n} \Delta t
$$

Then we propose to model the right handside of (4.3) by a random variable $E^{\prime}$-valued such that

$$
\mathbb{E}\left[\Delta V_{n}\right] \stackrel{\text { def }}{=} \mathbb{E}\left[\alpha_{n}\right]
$$

Here we consider also that the density (more exactly $\frac{1}{\rho}$ ) is included in the term $\alpha_{n}$. Our filter will have to learn this random variable $\alpha_{n}$ using the observation process.

Back to the continuous model we remark that $\mathbb{E}\left[\mathrm{d} V_{t} \mathrm{~d} V_{t}\right]=C_{0} \varepsilon_{t} \mathrm{~d} t$, and we propose another model for $\varepsilon_{n}$ taking the expectation of a random variable (yet denoted) $\varepsilon_{n}$ :

$$
\mathbb{E}\left[\varepsilon_{n}\right] \stackrel{\text { def }}{=} \frac{\mathbb{E}\left[\Delta V_{n} \Delta V_{n}\right]}{C_{0} \Delta t}
$$


By our models for the two commands, there will be some errors on the velocity representation, then we have to add a random error $\Delta W_{n}^{X}$ on the location equation. Thus the discrete adapted Pope model is the system:

$$
\left\{\begin{aligned}
X_{n+1} & =X_{n}+V_{n} \Delta t+\sigma_{n}^{X} \Delta W_{n}^{X} \\
V_{n+1} & =V_{n}+\mathbb{E}\left(\alpha_{n}\right)+C_{1} \frac{\mathbb{E}\left(\varepsilon_{n}\right)}{k_{n}}\left[V_{n}-\Pi_{n}^{\delta}\left(V_{n}\right)\left(X_{n}\right)\right] \Delta t+\sqrt{C_{0} \mathbb{E}\left(\varepsilon_{n}\right)} \Delta W_{n}^{V} .
\end{aligned}\right.
$$

If we use this model without any care, the incompressible hypothesis is disrupted, but using the filtering method and observation of incompressible fluids, we hope that this quality will be restored.

Now, the system (4.4) has to be modified to express the coupling between the Lagrangian Acquisition system and the Observation Acquisition by a conditioning of the probability laws.

This coupling is a new closure method and we think with this technique to improve the accuracy and the computational performance of the filtering. Indeed, as seen in Section 3, when we condition a Markov kernel by observations, we reduce the number of particles necessary to filter the mean-field process. In return, the accuracy will be very linked to a good representation of the observation process.

Let us formalize the physical situation to be closer to the filtering problem. The system (4.4) can be rewritten in the dynamical system:

$$
\mathcal{X}_{n+1}=\mathcal{X}_{n}+b\left(\mathcal{X}_{n}, \hat{\eta}_{n}\right) \Delta t+\sigma\left(\mathcal{X}_{n}\right) \Delta W_{n}
$$

where $\mathcal{X}_{n}=\left(X_{n}, V_{n-1}, V_{n}\right)$ is the conditional mean-field fluid-signal and $\hat{\eta}_{n}$ is probability law of the filter, $\hat{\eta}_{n}=\operatorname{Law}\left(\mathcal{X}_{[0, n]} \mid Y_{[0, n]}\right), b$ is a function with the hypotheses of Section 3 and a Lipschitz constant depending on $\delta$. In this model $\sigma$ is a function of the state vector $\mathcal{X}_{n}$. We have to assume that this is a positive, non-zero and bounded function. This model is more general than the system (3.2). But with the hypotheses, the control is easy to obtain (see [1], Thm. 6.5.1). In the physical applications, this hypothesis relative to the finite energy of the physical system will be insure. This is our stochastic model and we have to use it to filter the measurements of a turbulent fluid.

We consider the perturbed observations of the velocities $Y_{n}=H\left(\mathcal{X}_{n}\right)+\sigma_{n}^{Y} W_{n}$ where $\sigma_{n}^{Y}$ is still a bounded positive constant, and the filtering problem is to compute the $\operatorname{Law}\left(\mathcal{X}_{[0, n]} \mid Y_{[0, n]}\right)$.

$\mathcal{X}_{n}$ is then a conditional Markov process with the transition law $M_{n+1, \hat{\eta}_{n}}$ where

$$
M_{n+1, \hat{\eta}_{n}}((x, u), d(z, v))=\mathbb{P}^{\mathcal{X}_{n+1} \mid \mathcal{X}_{n}, \mathcal{Y}_{0}^{n}}(d(z, v) \mid(x, u))
$$

Section 3 claims that the particle approximation requires a system of $N$ particles and provides an error in $\mathcal{O}\left(\frac{1}{\sqrt{N}}\right)$.

In its spread out form the fluid-system is:

$$
\left\{\begin{aligned}
X_{n+1}= & X_{n}+V_{n} \Delta t+\sigma_{n}^{X} \Delta W_{n}^{X} \\
V_{n+1}= & V_{n}+\mathbb{E}\left(\alpha_{n} \mid Y_{0} \ldots Y_{n}\right) \Delta t \\
& -C_{1} \frac{\mathbb{E}\left(\varepsilon_{n} \mid Y_{0} \ldots Y_{n}\right)}{k_{n}}\left[V_{n}-\frac{\int v G^{\delta}\left(X_{n}-x\right) \mathbb{P}^{\left(X_{n}, V_{n} \mid Y_{0} \ldots Y_{n}\right)}(d(x, v))}{\left.\int G^{\delta}\left(X_{n}-x\right) \mathbb{P}^{\left(X_{n}, V_{n} \mid Y_{0} \ldots Y_{n}\right)(d(x, v))}\right] \Delta t}\right. \\
& +\sqrt{C_{0} \mathbb{E}\left(\varepsilon_{n} \mid Y_{0} \ldots Y_{n}\right)} \Delta W_{n}^{V} \\
Y_{n}= & H\left(V_{n}\right)+\sigma^{Y} W_{n}^{Y}
\end{aligned}\right.
$$

with $k_{n}=\frac{1}{2} \int\left[w-\frac{\int v G^{\delta}(z-x) \mathbb{P}^{\left(X_{n}, V_{n} \mid Y_{0} \ldots Y_{n}\right)}(d(x, v))}{\int G^{\delta}(z-x) \mathbb{P}^{\left(X_{n}, V_{n} \mid Y_{0} \ldots Y_{n}\right)}(d(x, v))}\right]^{2} \mathbb{P}^{\left(X_{n}, V_{n} \mid Y_{0} \ldots Y_{n}\right)}(d(z, w))$

Now we have to add the localization step to couple the system with the Acquisition Path of the sensor. This second coupling means that the different conditional expectation and the Eulerian average $\langle$.$\rangle are in fact$ approximated by $\mathbb{E}\left(. \mid X_{[0, t]} \in B_{t}^{\varepsilon}\left(Z_{[0, t]}\right), Y_{t}\right)$. This third part of the algorithm is an acceptation/rejection step with a redistribution in the set of balls $B_{t}^{\varepsilon}\left(Z_{[0, t]}\right)$.

For 3D atmospheric flows, the SLM model made for homogeneous flows is inappropriate and we suggest to build a model following the proposition of Das and Durbin (see [8]) for dispersion in a stratified turbulent flow. 
Our stochastic model can be seen as the dispersion of a fluid particle with respect to a virtual element following the mean flow:

$$
\left\{\begin{aligned}
\mathrm{d} X_{t}= & V_{t} \mathrm{~d} t \\
\mathrm{~d} V_{h, t}= & -\nabla_{h}\langle p\rangle \mathrm{d} t-\frac{C_{1}}{2} \frac{\varepsilon_{t}}{k_{t}}\left(V_{h, t}-\langle V\rangle_{h, t}\right) \mathrm{d} t \\
& +\left(C_{2 \theta}-1\right)\left(W_{t}-\langle W\rangle_{t}\right) \frac{\mathrm{d}\langle V\rangle_{h, t}}{\mathrm{~d} z} \mathrm{~d} t+\left(C_{0} \varepsilon_{t}\right)^{\frac{1}{2}} \mathrm{~d} B_{t}^{V_{h}} \\
\mathrm{~d} W_{t}= & \mathrm{d}\langle W\rangle_{t}-\frac{C_{1}}{2} \frac{\varepsilon_{t}}{k_{t}}\left(W_{t}-\langle W\rangle_{t}\right) \mathrm{d} t \\
& +\left(1-C_{5 \theta}\right) \beta_{t} g\left(\theta_{t}-\langle\theta\rangle_{t}\right) \mathrm{d} t+\left(C_{0} \varepsilon_{t}\right)^{\frac{1}{2}} \mathrm{~d} B_{t}^{W} \\
\mathrm{~d} \theta_{t}= & \mathrm{d}\langle\theta\rangle_{t}-\left(C_{1 \theta}-\frac{C_{1}}{2}\right) \frac{\varepsilon_{t}}{k_{t}}\left(\theta_{t}-\langle\theta\rangle_{t}\right) \mathrm{d} t \\
& -\left(W_{t}-\langle W\rangle_{t}\right) \frac{\mathrm{d}\langle\theta\rangle_{t}}{\mathrm{~d} z} \mathrm{~d} t+\left(C_{\theta}\right)^{\frac{1}{2}} \mathrm{~d} B_{t}^{\theta}
\end{aligned}\right.
$$

where $V_{h, t}$ is the horizontal speed, $\nabla_{h}$ is horizontal gradient operator (the partial derivatives of the horizontal components), $W$ is the vertical speed, $\theta$ is the temperature and $B_{t}^{V_{h}}, B_{t}^{W}, B_{t}^{\theta}$ are independent Wiener processes.

The system (4.5), using the dispersion of a fluid particle with respect to the mean flow, is our first adjustment of the Das and Durbin model to be usable in our filtering problem. We add some simplifications particularly on the constants: $C_{0}$ becomes the universal Kolmogorov constant with $C_{0}=2.1$, and using the paper of Das and Durbin [8] we set the other constants to $C_{1}=1.8, C_{1 \theta}=2.5, C_{2 \theta}=0.6$ and $C_{5 \theta}=1 / 3, C_{\theta}$ will be learned as $\varepsilon_{t}$ can be.

The buoyancy coefficient $\beta_{t}$ is approximated by the reciprocal of the local mean temperature $\Pi_{t}^{\delta}\left(\theta_{t}\right)\left(X_{t}\right)$, $\beta_{t}=\frac{1}{\Pi_{t}^{\delta}\left(\theta_{t}\right)\left(X_{t}\right)}$. It remains hidden parameters, the vertical gradients $\Gamma_{t}^{h}=\frac{\mathrm{d}\left\langle V^{h}\right\rangle}{\mathrm{d} z}$ and $\Gamma_{t}^{\theta}=\frac{\mathrm{d}\langle\theta\rangle}{\mathrm{d} z}$, where $z$ is the vertical dimension. The particle filter will learn these gradients using the selected and well-adapted particles.

Applying the same methods used for the SLM model, we write:

$$
\begin{array}{rlrl}
A_{n}^{h} & =\mathbb{E}\left[V_{n}^{h}-V_{n-1}^{h}\right] & E_{n}^{h} & =\frac{\mathbb{E}\left[V_{n}^{h}-V_{n-1}^{h}\right]^{2}}{C_{0} \Delta t} \\
A_{n}^{W}=\mathbb{E}\left[W_{n}-W_{n-1}\right] & E_{n}^{W}=\frac{\mathbb{E}\left[W_{n}-W_{n-1}\right]^{2}}{C_{0} \Delta t} \\
A_{n}^{\theta}=\mathbb{E}\left[\theta_{n}-\theta_{n-1}\right] & C_{\theta}=\frac{\mathbb{E}\left[\theta_{n}-\theta_{n-1}\right]^{2}}{C_{0} \Delta t} .
\end{array}
$$

For the kinetic energy, we use different forms for the horizontal and the vertical components to restore the stratified medium: $k_{n}^{h}=\frac{1}{2} \Pi_{n}^{\delta}\left(\left[V_{n}^{h}-\Pi_{n}^{\delta}\left(V_{n}^{h}\right)\right]^{2}\right)\left(X_{n}\right)$ and $k_{n}^{W}=\frac{1}{2} \Pi_{n}^{\delta}\left(\left[W_{n}-\Pi_{n}^{\delta}\left(W_{n}\right)\right]^{2}\right)\left(X_{n}\right)$. For the temperature equation, we choose $k_{n}^{2}=\left(k_{n}^{h}\right)^{2}+\left(k_{n}^{W}\right)^{2}$ and $E_{n}^{2}=\left(E_{n}^{h}\right)^{2}+\left(E_{n}^{W}\right)^{2}$.

These models are made to learn from the observations the hidden parameters and close the system by conditioning the transition law. The dynamical system is therefore:

$$
\left\{\begin{aligned}
X_{n+1}= & X_{n}+V_{n} \Delta t+\sigma^{X} \Delta B_{n}^{X} \\
V_{n+1}^{h}= & V_{n}^{h}+A_{n}^{h}-\frac{C_{1}}{2} \frac{E_{n}^{h}}{k_{n}^{h}}\left[V_{n}^{h}-\Pi_{n}^{\delta}\left(V_{n}^{h}\right)\left(X_{n}\right)\right] \Delta t \\
& +\left(C_{2 \theta}-1\right)\left[W_{n}-\Pi_{n}^{\delta}\left(W_{n}\right)\left(X_{n}\right)\right] \Gamma_{n}^{h} \Delta t+\left(C_{0} E_{n}^{h}\right)^{\frac{1}{2}} \Delta B_{n}^{h} \\
W_{n+1}= & W_{n}+A_{n}^{W}-\frac{C_{1}}{2} \frac{E_{n}^{W}}{k_{n}^{W}}\left[W_{n}-\Pi_{n}^{\delta}\left(W_{n}\right)\left(X_{n}\right)\right] \Delta t \\
& +\left(1-C_{5 \theta}\right) \beta_{n} g\left[\theta_{n}-\Pi_{n}^{\delta}\left(\theta_{n}\right)\left(X_{n}\right)\right] \Delta t+\left(C_{0} E_{n}^{W}\right)^{\frac{1}{2}} \Delta B_{n}^{W} \\
\theta_{n+1}= & \theta_{n}+A_{n}^{\theta}-\left(C_{1 \theta}-\frac{C_{1}}{2}\right) \frac{E_{n}}{k_{n}}\left[\theta_{n}-\Pi_{n}^{\delta}\left(\theta_{n}\right)\left(X_{n}\right)\right] \Delta t \\
& -\left[W_{n}-\Pi_{n}^{\delta}\left(W_{n}\right)\left(X_{n}\right)\right] \Gamma_{n}^{\theta} \Delta t+\left(C_{\theta}\right)^{\frac{1}{2}} \Delta B_{n}^{\theta} \\
X_{0}= & x_{0} \\
V_{0}^{h}= & v_{0}, \quad W_{0}=w_{0} \\
\theta_{0}= & \theta_{0} .
\end{aligned}\right.
$$


We have seen previously that the Markov kernels have to be conditioned to the path, this has a consequence for the dynamic model. Indeed denoting $V_{n}^{A}$ the Acquisition velocity (velocity of the fluid of along the Acquisition Path), $V_{n}^{Z}$ the course velocity along the Acquisition Path, and $V_{n}^{L}$ the velocity of the Lagrangian fluid particle following (4.6) using the implicit derivation:

$$
V_{n+1}^{A}=V_{n+1}^{L}+G_{n, Z}^{A} V_{n}^{Z} \Delta t+\sigma^{X} G_{n, Z}^{A} \Delta B_{n}^{X}
$$

where $G_{n, Z}^{A}$ is the gradient of fluid velocity along the Acquisition Path. In the case of a particle resolution of the system, $G_{n, Z}^{A}$ is approached by $\hat{G}_{n, Z}^{A, N}$ the empirical gradient in the direction $\left[Z_{n}-Z_{n-1}\right]$ computed using the selected particles.

The Markov kernel associated to (4.7) is denoted $M_{n+1, \hat{\eta}_{n}}^{B}$. In 1D flow (resp. 3D flow) we consider $\mathcal{X}_{n}=\left(X_{n}, V_{n}\right)$ (resp. $\mathcal{X}_{n}=\left(X_{n}, V_{n}^{h}, W_{n}, \theta_{n}\right)$ ). The superscript $B_{n}$ means 'conditioned to be in the ball $B_{n}^{\varepsilon}\left(Z_{n}\right)^{\prime}$. The discrete filtering algorithm is then described by the following sequence:

$$
\mathcal{X}_{n}^{B_{n}} \stackrel{\text { w.r.t. } S_{n, \eta_{n}}}{\longrightarrow} \hat{\mathcal{X}}_{n}^{B_{n}} \stackrel{\text { w.r.t. } M_{n+1, \hat{\eta}_{n}}^{B}}{\longrightarrow} \tilde{\mathcal{X}}_{n+1} \stackrel{\text { w.r.t } \mathcal{S}_{n+1, \tilde{\eta}_{n+1}}^{Z, B}}{\longrightarrow} \mathcal{X}_{n+1}^{B_{n+1}}
$$

where $\tilde{\eta}_{n+1}=\operatorname{Law}\left(\tilde{\mathcal{X}}_{n+1} \mid \mathcal{X}_{[0, n]} \in B_{n}^{\varepsilon}\left(Z_{[0, n]}\right), Y_{[0, n]}\right)$.

These two examples of adaptation of turbulent fluid models are necessary to filter simulated or real data in 1D, 2D or 3D atmospheric wind. Others models or parameterizations or learning techniques are possible. In the next section we will use the 3D stratified model (4.6) to filter real data.

\subsection{Application to $3 \mathrm{D}$ real wind measurements}

We have described all the algorithms for the various parts of the problem put down by the filtering of the mobile measurements of a fluid.

It is necessary to test our methods on simulated or real data. To check the ability of our filter to perform good estimations, we propose to use a reference signal, real or not. We perturb this signal with a random noise using simple or complex probability laws. Then, our algorithms are used to deliver a filtered signal. By this procedure, we are able to compare the filtered signal with the initial reference signal. The use of real data requires some precautions especially on the high quality of the measurements to be considered as a reference.

We have to interpret the results with care. The reference signal is one realization of the random medium taking into account its parameters, the filtered signal is built with the estimated parameters of the observed medium. So, there is a natural difference between the two series, but they have to give the same turbulence characteristics particularly on large scale and in their energetic cascades, seen with their Power Density Spectra (PSD).

The quality of the estimations have to be evaluated with several manner. The first one is the filtering of fluid velocities simulated data. This examination enables to test the sequences of the algorithms. We do not present these results on 1D or 2D simulated fluids and prefer to examine here directly the capacity of the method with real 3D flows.

Real or simulated, the first comparison will be visual with the examination of their filtered and reference series and the PSD. We remind that atmospheric turbulent flows, in the domain of our measurements, follow the empirical Kolmogorov law (K41) (see for example Frisch's book [10]). In log-log diagram, the PSD have to follow a slope in $-5 / 3$ and we expect this kind of energetic cascade for the reference signal and for the filtered one. Another possible control of the filter quality can be the calculation of some large scale classical turbulence indicators. We will present these calculations in a further publication.

Here, we present the results of filtering using atmospheric data recorded at $5 \mathrm{~Hz}$ with an ultrasonic anemometer in the experimental field of the French Weather Service Research Center in Toulouse, France, at two moments: first on the 12th of May 2006 between $12 \mathrm{~h} 03$ and 12h07 UTC and second on the 14th of June 2006 between $15 \mathrm{~h} 33$ and $15 \mathrm{~h} 37 \mathrm{UTC}$. 

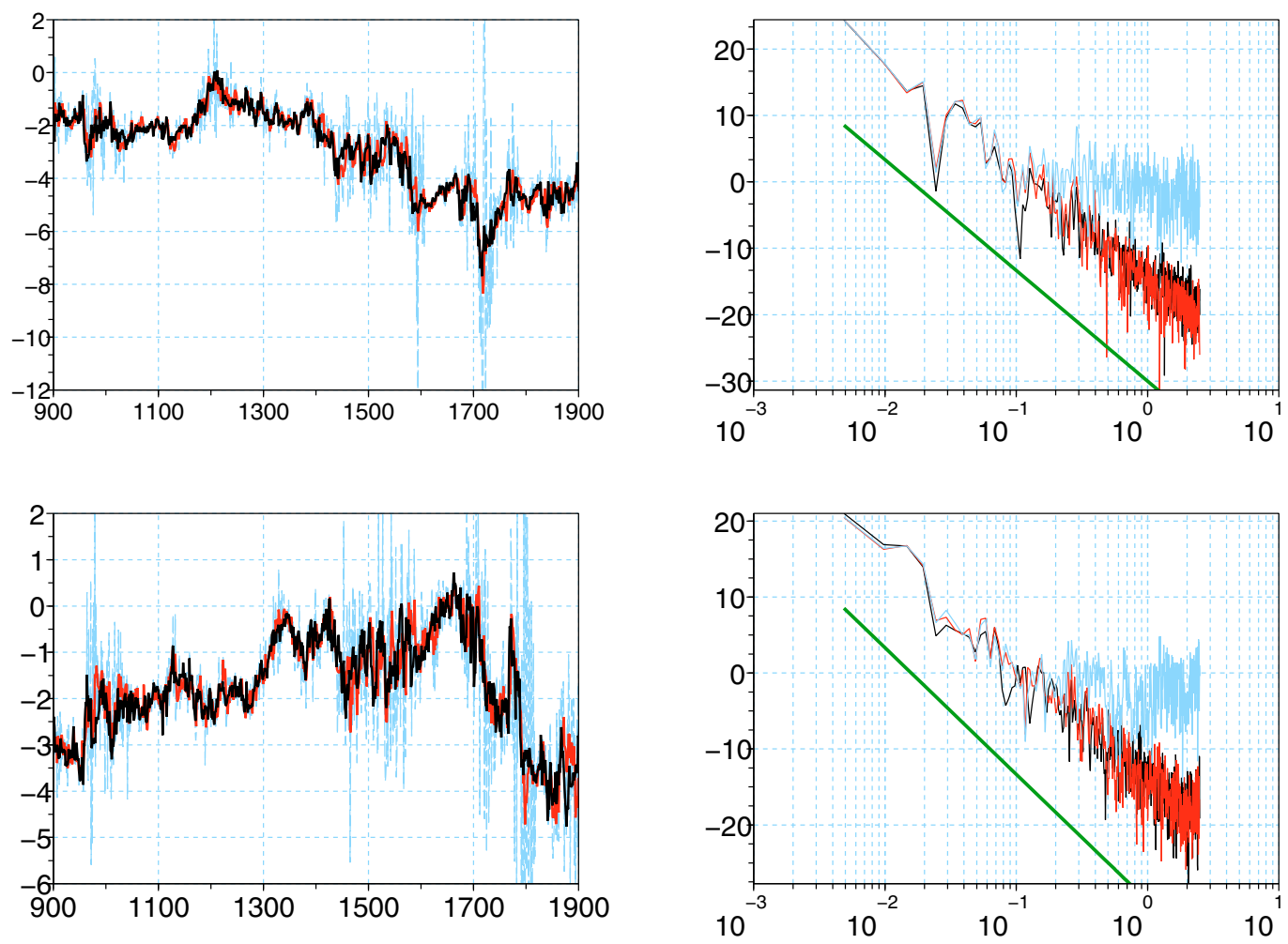

FIGURE 4. Left, series of horizontal wind (velocity $\left(\mathrm{m} \cdot \mathrm{s}^{-1}\right)$ vs. time step number) and right, PSD (with a log-log scale, power $(\mathrm{dB})$ vs. frequency $(\mathrm{Hz})$ ). The series on the top is the $U$ component and the bottom the $V$ component. Time series are taken the 12th of May 2006 between $12 \mathrm{~h} 03$ and 12h07 UTC. In light blue the perturbed signal to be denoised, in black the reference signal and in red the filtered with 800 particles. (Figure in color available online at www. esaim-m2an.org/.)

The choice of this two dates and the hours is only determined by the measurement quality. The ultrasonic anemometer gives a measure of the sonic temperature, which is converted in a real temperature with thermodynamics laws. We choose this method of measurement for the temperature to insure a colocalization of all the measurements (wind and temperature). But it is well known that ultrasonic anemometer are far from being the best thermometer. So the temperature signal is taken as reference but it is a stopgap. For further experimental campaigns we will add to the anemometer a thin wire thermometer to measure a fast temperature.

In the two cases we choose to add a random noise built using the local empirical variance of the reference signals and a Gaussian law. This noise is an upper limit for turbulence, and there is a memory effect which can give dramatic errors if the filter does not return the right parameter of the fluid. Sometimes our noise is quite big, barely too strong, but it provides a good test on the robustness of our filter.

For the first experiment on 12th of May, Figures 4 (horizontal wind) and 5 (vertical wind and temperature) show in light blue dash line the noisy signal of the three components of wind and temperature, the black line is the signal of reference to retrieve. The filter uses the algorithm described in the previous sections with 800 particles for the approximation. The red signal is the output of our filter using a SCILAB code.

Even if the perturbations are strong, the filtered signal compares well with the reference component. On the diagram of PSD, the correction is very sharp, with a decreasing of power following the reference spectrum, 

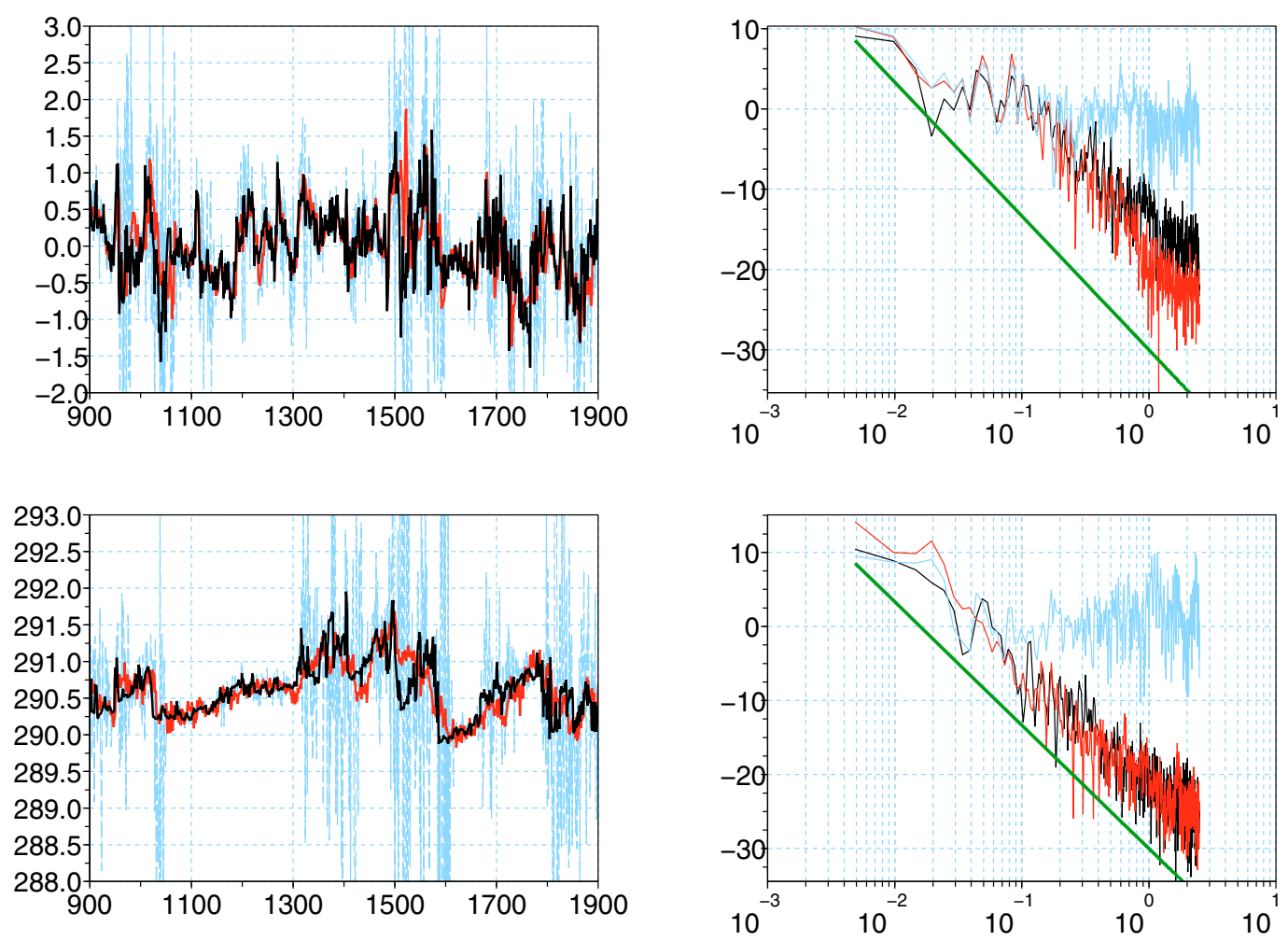

FiguRE 5. Series ( $x$-axis is time step number). On the top, the vertical velocity $\left(\mathrm{m} \cdot \mathrm{s}^{-1}\right)$, on the bottom, the temperature $(\mathrm{K})$ and PSD (power $(\mathrm{dB})$ vs. frequency $(\mathrm{Hz})$ ). Time series are taken the 12th of May 2006 between $12 \mathrm{~h} 03$ and 12h07 UTC. In light blue the perturbed signal to be denoise, in black the reference signal and in red the filtered with 800 particles. (Figure in color available online at www.esaim-m2an.org/.)

far from the perturbed signal. In detail, there are slight differences between the filtered and the reference signals, it comes from our method itself. Indeed, the algorithm estimates the characteristic parameters of the fluid through the model used by the filter, and returns a filtered signal, while the output is one possible realization of the medium. This is the reason why the spectral correction is very good, while the series show differences. In conclusion, the noise is entirely subtracted out of the perturbed signal.

Our method, which estimates some characteristic terms, retrieves also turbulence parameters at high frequency which are accessible by the prediction model. It could be possible to evaluate the turbulent dissipation rate or buoyancy coefficient or 3-d gradients of Eulerian averages, etc., and of course the Acquisition Path of the measurement sensors. Figure 6 gives an example of the series of turbulence dissipation rates and the vertical gradient of temperature. These estimations with high frequency of turbulent quantities are something very new. Usually in meteorological sciences these parameters are estimated with long time series, empirical means and doubtful ergodic hypothesis. Our method provide estimations of these quantities at the same frequency as the measurements and a real sense for the Eulerian averages.

This result is very promising but we do not have to forget that the filtered signal is very dependent on the chosen model. If the model is not pertinent relatively of the dynamics of the random medium, it could have some disturbances, sometimes annoying. To illustrate this fact, we can present an experiment where the filtered temperature signal has a poor quality. Figures 7 (horizontal wind) and 8 (vertical wind and temperature) show, 

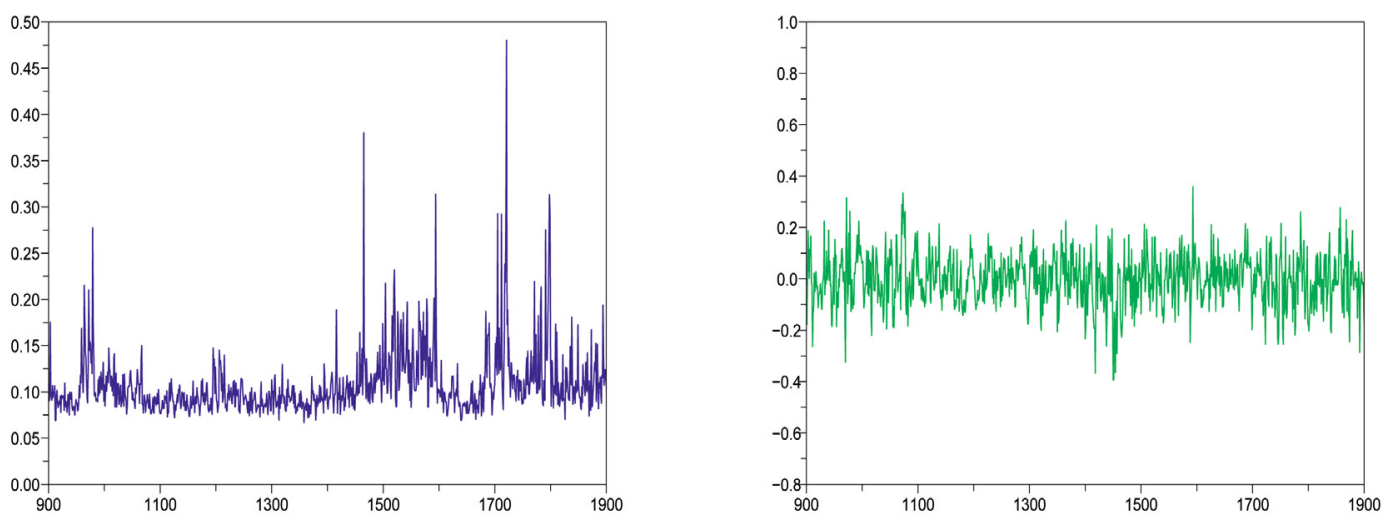

FiguRE 6. Left, estimation of $\varepsilon_{n}$ parameter $\left(\mathrm{m}^{2} \cdot \mathrm{s}^{-3}\right)$ and right gradient of mean temperature $\left(\mathrm{K} \cdot \mathrm{m}^{-1}\right)$ with 800 particles. Time series are taken the 12 th of May 2006 between $12 \mathrm{~h} 03$ and $12 \mathrm{~h} 07$ UTC. $X$-axis is time step number.
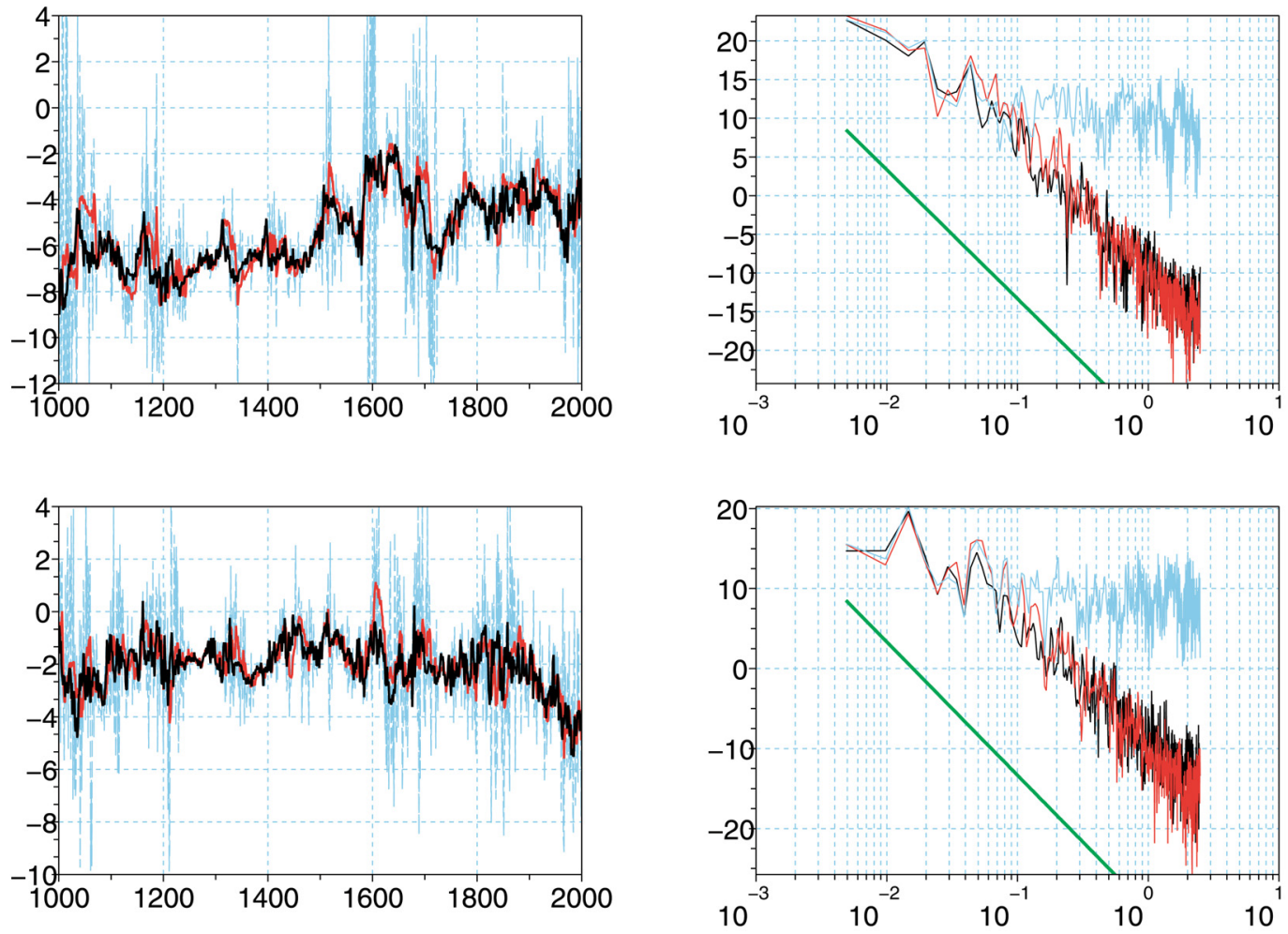

FIgURE 7. Left, series of horizontal wind (velocity $\left(\mathrm{m} \cdot \mathrm{s}^{-1}\right)$ vs. time step number) and right, PSD (with a log-log scale, power $(\mathrm{dB})$ vs. frequency $(\mathrm{Hz})$ ). The series on the top is the $U$ component and the bottom the $V$ component. Time series are taken the 14th of June 2006 between $15 \mathrm{~h} 33$ and $15 \mathrm{~h} 37$ UTC. In light blue the perturbed signal to be denoise, in black the reference signal and in red the filtered with 800 particles. (Figure in color available online at www.esaim-m2an.org/.) 

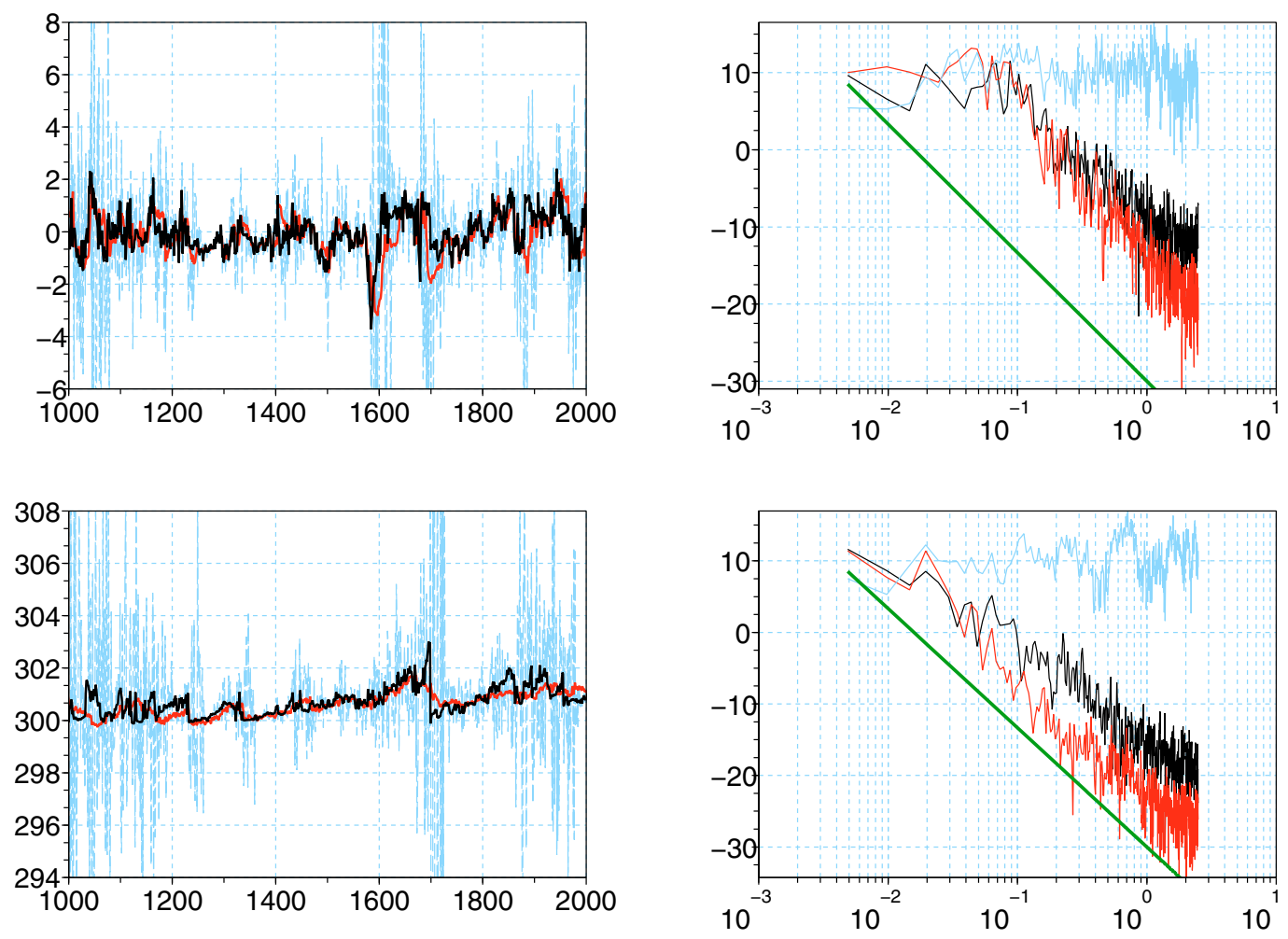

FiguRE 8 . Series ( $x$-axis is time step number). On the top, the vertical velocity $\left(\mathrm{m} \cdot \mathrm{s}^{-1}\right)$, on the bottom, the temperature $(\mathrm{K})$ and PSD (power $(\mathrm{dB})$ vs. frequency $(\mathrm{Hz})$ ). Time series are taken the 14th of June 2006 between $15 \mathrm{~h} 33$ and 15h37 UTC. In light blue the perturbed signal to be denoise, in black the reference signal and in red the filtered with 800 particles. (Figure in color available online at www.esaim-m2an.org/.)

with the same color code, the three signals (noised, filtered and of reference) for the second date on the 14 th of June 2006 at $15 \mathrm{~h} 30$ UTC. The (horizontal or vertical) wind is always properly filtered, but the temperature response is not correct as indicates by the PSD.

There is two leads. The first one is the relevance of the model for this specific real meteorological situation. The second is the quality on the measurement used as reference.

In the stratified Lagrangian model, the four components are coupled and the filtering of the wind is dependent on a good estimation of the temperature. But the three wind components behave correctly, while the temperature signal seems very different from the reference. At this point there is no unique answer, the problem could be on the measurement of temperature, or in the choice of the model or its adaptation or may be there is a specific behaviour of the atmosphere not taken into account by the model (water vapor, water content, etc.). To insure that the problem does not come from too big numerical perturbations, we have proceeded with various level of noises without any improvement of the filtered temperature structure. The conclusion could be that the dynamics of the reference temperature and the dynamics of the temperature deduced by the model are different, while the $3 \mathrm{D}$ wind is correctly represented, but we have no argument yet to say if the problem lies in the chosen reference for temperature.

For all the distinct experiments we have made, with various regime of wind, period of the year, hour of the day, etc., in the majority of the cases the filtering is good with an accurate correction. Nevertheless, when the atmospheric turbulence is too weak (early morning, no wind situation, etc.), the filtered signal remains 
too turbulent just because the laminar regime is not well fitted by our Lagrangian models. Out the case of laminar flows, sometimes there are some problems with the temperature filtering while the wind is accurately estimated. The situation shown previously, the 14th of June 2006, is an example of this fact. For the moment, there exists no satisfying explanations. It illustrates the difficulties of this exercise in real conditions and gives some clues for the further experiments and improvements.

\section{Outcomes And FURTher DEVELOPMENTS}

In this paper, we have described how to filter perturbed observations of a random medium taken along a random path and applied the result to real meteorological data. First, we define precisely the Acquisition Process as the statement along a path of a random medium. We provide some examples of use and describe an algorithm of estimation in the case of homogeneous medium represented by its Lagrangian flows. Subsequently we have developed new algorithms for the filtering of mean-field processes and suggest particle approximations. We give their asymptotic behaviour when the number of particles goes to infinity. Then to apply to fluid velocities measurements we have adapted some existing physical models to be able to learn turbulence parameters. These adaptations correspond to the coupling of the model with the Acquisition Path and the Observational Acquisition Process. We have seen that these couplings with a conditioning of the model to the observations are a new manner to close a dynamical system.

For the physical applications, we have chosen two real meteorological situations. We have corrupted the reference signal with colored Gaussian noises, occasionally with very strong perturbations. Our filter provides quite good estimations with pertinent series of filtered signals and a sharp correction of the energetic cascades. High frequency estimations for turbulent parameters with a sense of the Eulerian means are an important innovation given by the Acquisition Process and stochastic filtering for mean-field processes.

But nonlinear filtering never lives in a perfect world and we have seen that problems may occur especially with laminar flows or in the treatments of the temperature. About the temperature, the question of the pertinence of ultrasonic anemometer for the measurements or the pertinence of our stochastic model for temperature component had not be decided.

But this work is a first stage. For atmospheric data assimilation we have successfully used the Acquisition Process to enhance the performance of an Ensemble Kalman Filter with a coupled Local Particle Filter (see the short report of Baehr and Pannekoucke [3]). We hope to use in future developments these different techniques to estimate turbulent parameters for high resolution atmospheric forecasting model assimilation.

Using the Acquisition Process and the data fusion particle techniques, it is also our intention to contribute to the design of new integrated systems for airborne turbulence measurements and with a more physical modeling to filter numerically more complex atmospheric set of parameters including water content, chemical or aerosol concentration, droplets counting, etc. Theoretical works are also in progress to modify the algorithm presented here and not break the incompressibility hypothesis like the modification of the model proposed here does.

Back to the mathematical part of this work, there is also some study to complete the understanding of the filter based on Acquisition Process and particularly to characterize the closure of a model by observations. The article of Baehr and Pannekoucke [3] explain that in high dimension genetic filtering algorithms require a critical number of particles to insure the non-divergence of the filter. We have to deal studies about this aspect and propose new filter coupling various nonlinear filters and separating the treatment of linear and nonlinear components of a system and find strategies of piloting/tuning of the selection parameters to be adapted to models with large degrees of freedom. Local Particle Filter with Acquisition Process presented here should be a partial answer.

Acknowledgements. The author thanks the referee for his very careful review and comments that include many points and improve significantly the clarity of this paper. 


\section{REFERENCES}

[1] C. Baehr, Modélisation probabiliste des écoulements atmosphériques turbulents afin d'en filtrer la mesure par approche particulaire. Ph.D. Thesis University of Toulouse III - Paul Sabatier, Toulouse Mathematics Institute, France (2008).

[2] C. Baehr and F. Legland, Some Mean-Field Processes Filtering using Particles System Approximations. In preparation.

[3] C. Baehr and O. Pannekoucke, Some issues and results on the EnKF and particle filters for meteorological models, in Chaotic Systems: Theory and Applications, C.H. Skiadas and I. Dimotikalis Eds., World Scientific (2010).

[4] G. BenArous, Flots et séries de Taylor stochastiques. Probab. Theor. Relat. Fields 81 (1989) 29-77.

[5] M. Bossy and D. Talay, Convergence rate for the approximation of the limit law of weakly interacting particles. 2: Application to the Burgers equation. Ann. Appl. Prob. 6 (1996) 818-861.

[6] B. Busnello and F. Flandoli and M. Romito, A probabilistic representation for the vorticity of a 3d viscous fluid and for general systems of parabolic equations. Proc. Edinb. Math. Soc. 48 (2005) 295-336.

[7] P. Constantin and G. Iyer, A stochastic Lagrangian representation of 3-dimensional incompressible Navier-Stokes equations. Commun. Pure Appl. Math. 61 (2008) 330-345.

[8] S. Das and P. Durbin, A Lagrangian stochastic model for dispersion in stratified turbulence. Phys. Fluids 17 (2005) 025109.

[9] P. Del Moral, Feynman-Kac Formulae, Genealogical and Interacting Particle Systems with Applications. Springer-Verlag (2004).

[10] U. Frisch, Turbulence. Cambridge University Press, Cambridge (1995).

[11] G. Iyer and J. Mattingly, A stochastic-Lagrangian particle system for the Navier-Stokes equations. Nonlinearity 21 (2008) $2537-2553$.

[12] I. Karatzas and S.E. Shreve, Brownian Motion and Stochastic Calculus. Springer-Verlag (1988).

[13] S. Méléard, Asymptotic behaviour of some particle systems: McKean Vlasov and Boltzmann models, in Probabilistic Models for Nonlinear Partial Differential Equations, Lecture Notes in Math. 1627, Springer-Verlag (1996).

[14] R. Mikulevicius and B. Rozovskii, Stochastic Navier-Stokes Equations for turbulent flows. SIAM J. Math. Anal. 35 (2004) $1250-1310$.

[15] S.B. Pope, Turbulent Flows. Cambridge University Press, Cambridge (2000).

[16] A.S. Sznitman, Topics in propagation of chaos, in École d'Eté de Probabilités de Saint-Flour XIX-1989, Lecture Notes in Math. 1464, Springer-Verlag (1991). 